\title{
Les stations mésolithiques du Raumarais, commune de Digulleville (Manche)
}

Léonard Cuffez, Frédérik Letterlé, Philippe Amourette, Philippe Sabatier, Christine Thauvin et Yves Vaganay

\section{(2) OpenEdition}

12 Journals

Édition électronique

URL : http://journals.openedition.org/rao/189

DOI : 10.4000/rao.189

ISBN : 978-2-7535-1606-9

ISSN : $1775-3732$

Éditeur

Presses universitaires de Rennes

Édition imprimée

Date de publication : 30 décembre 2006

Pagination : 151-169

ISBN : 978-2-7535-0574-2

ISSN : 0767-709X

\section{Référence électronique}

Léonard Cuffez, Frédérik Letterlé, Philippe Amourette, Philippe Sabatier, Christine Thauvin et Yves Vaganay, "Les stations mésolithiques du Raumarais, commune de Digulleville (Manche) », Revue archéologique de l'Ouest [En ligne], 23 | 2006, mis en ligne le 30 décembre 2008, consulté le 03

décembre 2020. URL : http://journals.openedition.org/rao/189 ; DOI : https://doi.org/10.4000/rao.189 


\title{
Les stations mésolithiques du Raumarais, commune de Digulleville (Manche)
}

\author{
Léonard Cuffez*, Frédérik Letterlé** et Philippe Amourette*** \\ avec la collaboration de Philippe Sabatier, Christine Thauvin et Yves Vaganay
}

Résumé : À la suite d'un projet de construction d'une nouvelle usine dans le centre atomique de La Hague, une opération de sauvetage archéologique fut entreprise en 1980. Deux gisements mésolithiques ont été reconnus (locus 1 et 3). La fouille du locus 3 a permis la découverte d’un foyer en cuvette. L'analyse du matériel lithique recueilli dans son comblement et ses abords nous incite à placer cette série au début du stade moyen du Mésolithique. L'outillage commun est caractérisé par un taux élevé de petits grattoirs. Les lamelles étroites à bord abattu, dont quelques exemplaires sont tronqués, prédominent les armatures à plus de $80 \%$. Ce taux n’a pas été confirmé pour l'instant sur d'autres sites du nord-Cotentin.

Abstract: The Mesolithic sites of Le Raumarais, commune of Digulleville (Manche)

As a result of the construction of a new building at the Hague Nuclear Centre, archaeological investigation was conducted in 1980. Two mesolithic sites (locus 1 and 3) were identified. Excavation on locus 3 revealed a hearth. Analysis of the lithic artefacts found in this and in the vicinity suggest that the Digulleville assemblage should be dated to the beginning of the middle Mesolithic period. Common tools include a high proportion of small scrapers and narrow backed bladelets with some truncated examples, where points represent more than $80 \%$ of the total. This ratio has not been yet observed at other sites of North Cotentin.

Mots clés : Manche, fouille, foyer, industrie lithique, Mésolithique moyen.

Key words: Manche, excavation, hearth, lithic industry, middle Mesolithic.

\section{INTRODUCTION}

Le site du Raumarais est localisé sur la commune de Digulleville (Manche), dans l'enceinte de l'usine de retraitement de déchets nucléaires de La Hague, à la pointe nordouest de la presqu'île du Cotentin (fig. 1). Il se trouve au centre d'un plateau où affleurent les grès feldspathiques de Herquemoulin (Cambrien), le grès armoricain (Arénigien) et les schistes de Beaumont (Llanvirnien), parfois recouverts de limons quaternaires.

\section{Historique}

Les stations ont été découvertes en 1975 à l'occasion de prospections réalisées par Y. Roupin et J. Flaman-Ruet (Verron 1975). À la suite d'un projet d'extension de l'usine, une fouille de sauvetage fut entreprise en 1980 sous la responsabilité de M. Philippe Amourette sur les locus 1 et 3 distants l'un de l'autre d'environ $150 \mathrm{~m}$ (fig. 2). Seule la fouille du locus 3 a révélé la présence de structures archéologiques (Verron 1981).

Le locus 2, qui correspondait à un habitat campaniforme, a été publié par ailleurs (Letterlé et Verron, 1986); quelques pièces, dont deux fragments de pointes à dos, indiquent que

* 44 avenue du Général de Gaulle, 97170 Le Perreux-sur-Marne. (leo.cuffez@free.fr)

** DRAC d'Auvergne, Hôtel de Chazerat, 4 rue Pascal, BP 378, 63010 Clermont-Ferrand Cedex 1. (frederik.letterle@culture.gouv.fr)

*** 562 rue Saint-Pierre, 76730 Aupegard. 


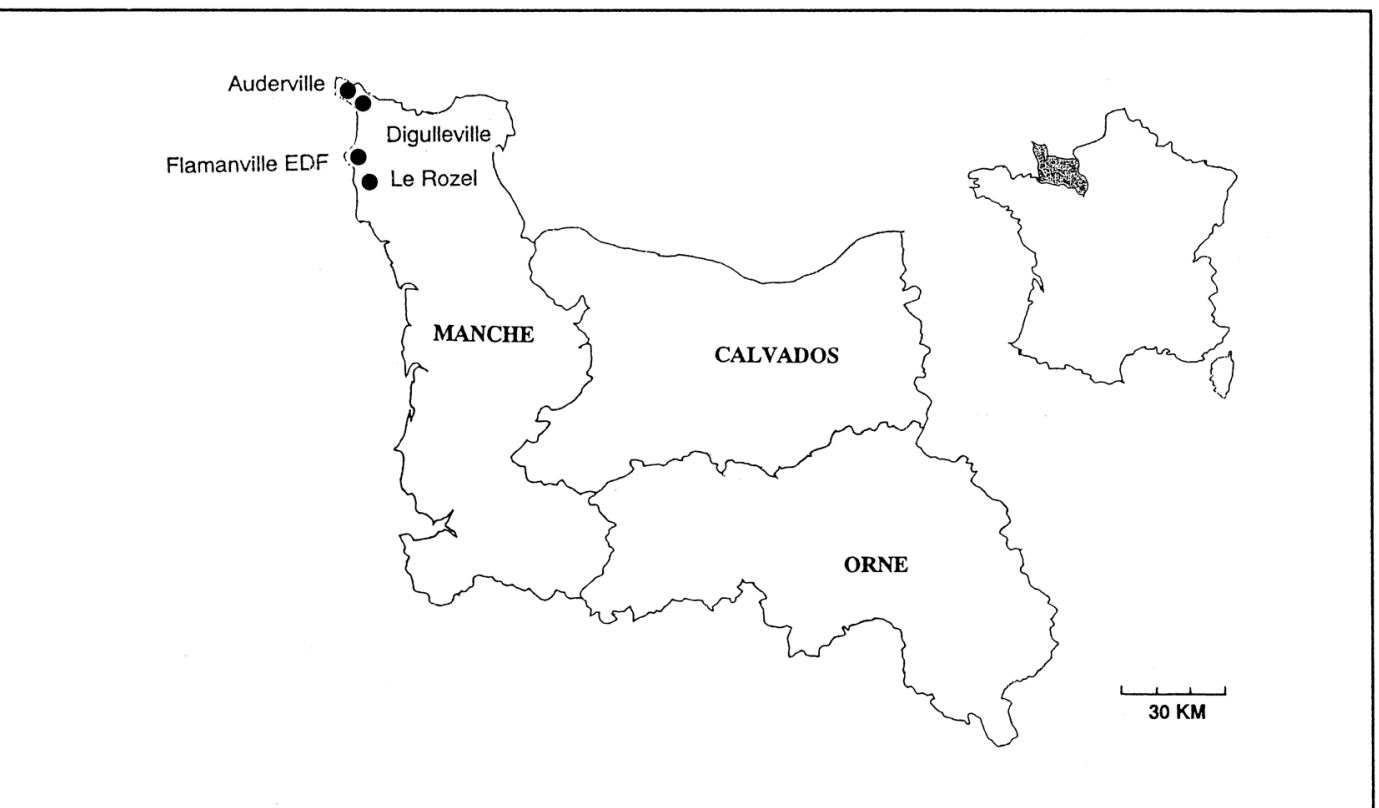

Figure 1 : Digulleville, «Le Raumarais » : carte de localisation.

Figure 1: Location map of the site.

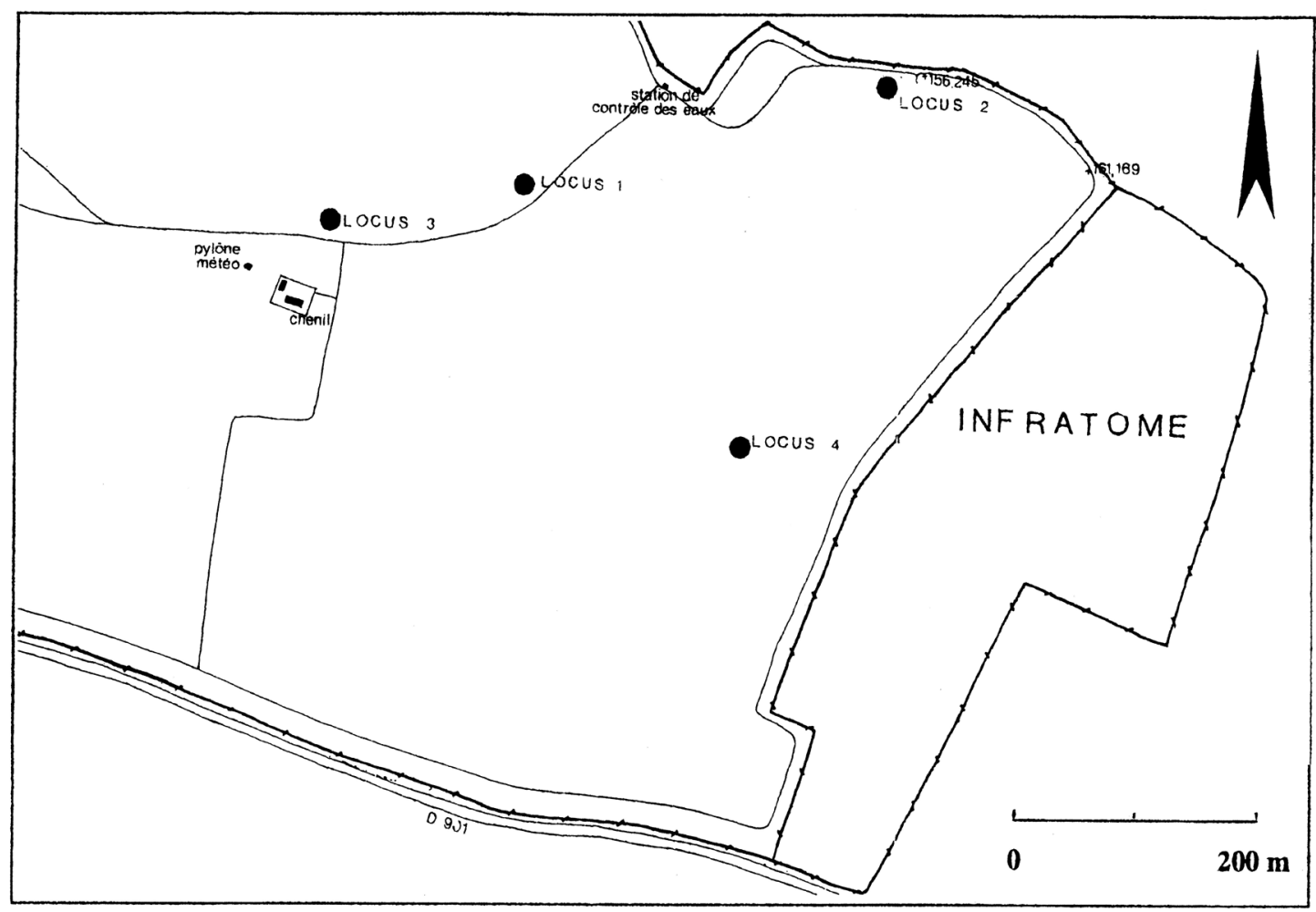

Figure 2 : Le Raumarais : plan de situation des gisements mésolithiques (locus 1 et 3), campaniforme (locus 2), et d'un gisement ayant fourni quelques éclats non datables (locus 4).

Figure 2: Location of the Mesolithic (locus $1 \& 3$ ) and Beaker (locus 2) deposits, plus another which yielded few undatable flakes. 
la zone occupée au Mésolithique se prolongeait jusque-là (soit environ $450 \mathrm{~m}$ ).

\section{LE LOCUS 1}

Il se situe en bordure ouest du chemin rural allant de la station météorologique à la station de contrôle des eaux de Sainte-Hélène (point-origine du carroyage métrique, coordonnées Lambert I : x 296,694 - y 228,405). La fouille a porté sur une trentaine de mètres carrés environ. La couche archéologique d'une épaisseur de $25 \mathrm{~cm}$, légèrement bioturbée, était située immédiatement au contact de l'horizon végétal.

\section{Le matériel brut}

Les inventaires ont été réalisés suivant les travaux publiés par le Dr J.-G. Rozoy (1967a et b, 1968a et b, 1971, 1978) et par le Groupe d'Étude de l'Épipaléolithique et du Mésolithique (GEEM, 1969, 1972, 1975). Les décomptes ont été effectués par niveau archéologique (tabl. 1).

\section{Matériau}

Seul le silex a été utilisé. L'examen des plages corticales conservées sur 79 éclats permet d'établir que $14 \%$ du débitage est réalisé sur galets probablement ramassés sur les plages voisines, $42 \%$ sur rognons partiellement roulés et $44 \%$ sur rognons non roulés dont l'origine reste à découvrir. Les artefacts ne comportent pas de patine, le silex gris clair domine avec $73 \%$ contre $27 \%$ pour le silex gris foncé.

\section{Produits de débitage}

Exception faite des nucléus, des déchets techniques (éclats d'avivage, chutes de burin, microburins) et des éclats inférieurs à $2 \mathrm{~cm}$ qui peuvent être considérés comme des éclats de retouches, les produits de débitage sont constitués principalement d'éclats (tabl. 2) et de lamelles.

\section{Module des enlèvements}

Les modules ont été calculés à partir des éclats entiers, suivant les rapports largeur / longueur publiés par A. LeroiGourhan (1966). Quatre éclats dont les bords étaient cassés n'ont pas été pris en compte (tabl. 3 et 4). Quatre modules se détachent assez nettement : éclats larges $(1 / 1)$ et très petits $(<2 \mathrm{~cm}): 19 \%$, éclats très larges $(>1)$ et très petits $(<2 \mathrm{~cm}): 16 \%$, éclats assez longs $(1 / 1,5)$ et petits $(2$ à $4 \mathrm{~cm}): 14 \%$, éclats larges $(1 / 1)$ et petits $(2$ à $4 \mathrm{~cm})$ : $11 \%$.

\section{Typologie du matériel}

- Les éclats : Ils présentent le plus souvent des talons lisses (49\%); les talons filiformes (18\%) et punctiformes (10\%) sont également bien représentés (fig. 3).

\begin{tabular}{|c|c|c|c|c|c|c|c|}
\hline Désignation & Surface & $\mathrm{C} / 1$ & $\mathrm{C} / 2$ & $2 / 3$ & $\mathrm{C} / 3$ & Nombre & $\begin{array}{c}\text { Total } \\
\text { partiel }\end{array}$ \\
\hline Nucléus unipolaires & & 2 & 3 & & & 5 & \\
\hline Nucléus pyramidaux & & & 1 & & & 1 & \\
\hline Nucléus prismatiques angulaires & & & & 1 & 1 & 2 & \\
\hline Nucléus informes & 2 & 1 & & & & 3 & 11 \\
\hline Éclat d'avivage & & & 1 & & & 1 & \\
\hline Chutes de burin & 1 & & & & & 1 & 2 \\
\hline Lames entières & & 1 & 1 & & & 2 & 2 \\
\hline Lamelles entières & 7 & 15 & 12 & & & 34 & \\
\hline Lamelles raccourcies & 1 & 7 & 6 & & & 14 & \\
\hline Corps de lamelles & 1 & 3 & 1 & & & 5 & \\
\hline Lamelles à bulbe enlevé & 2 & 4 & 1 & & & 7 & 60 \\
\hline Extrémités proximales de lamelles & 1 & 4 & 1 & & & 6 & \\
\hline Extrémités distales de lamelles & 1 & 2 & 1 & & & 4 & \\
\hline Débris de lamelles & & 1 & & & & 1 & 11 \\
\hline Microburins & 4 & 1 & & & & 5 & 5 \\
\hline Éclats non utilisés & 28 & 114 & 64 & 12 & 17 & 235 & \\
\hline Esquilles $(<1 \mathrm{~cm})$ & 8 & 37 & 11 & & 3 & 59 & \\
\hline Cassons & 5 & 13 & 3 & & 1 & 22 & 316 \\
\hline Total général & & & & & & & 407 \\
\hline
\end{tabular}

Tableau 1 : Le Raumarais, locus 1 : inventaire du matériel brut. Table 1: Le Raumarais, locus 1: distribution of unworked material. 


\begin{tabular}{|l|c|c|c|}
\hline \multicolumn{1}{|c|}{ Désignation } & Nombre & dont brûlés & Poids \\
\hline Éclats entiers & 116 & 7 & $549 \mathrm{~g}$ \\
\hline Éclats proximaux & 48 & 6 & 177 \\
\hline Éclats mésiaux & 33 & 13 & 33 \\
\hline Éclats distaux & 38 & 3 & 73 \\
\hline Cassons & 22 & 8 & 117 \\
\hline Esquilles $<1 \mathrm{~cm}$ & 59 & 16 & 16 \\
\hline
\end{tabular}

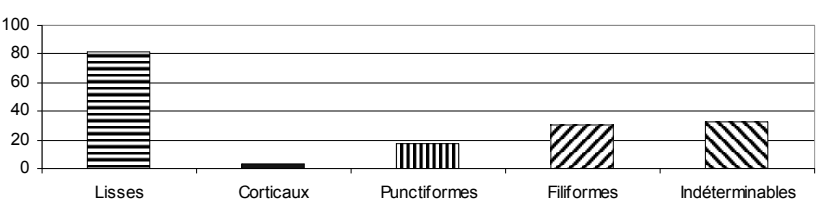

Tableau 2 : Le Raumarais, locus 1 : répartition des éclats.

Table 2: Le Raumarais, locus 1: distribution of flakes.

\begin{tabular}{|c|c|c|c|c|c|c|}
\hline Modules & Surface & $\mathrm{C} / 1$ & $\mathrm{C} / 2$ & $\mathrm{C} / 2-3$ & $\mathrm{C} / 3$ & Total \\
\hline Éclats très larges > 1 & 5 & 18 & 5 & 1 & 1 & 30 \\
\hline $\begin{array}{ll}\text { Éclats larges } & 1 / 1\end{array}$ & 5 & 13 & 15 & 7 & 1 & 41 \\
\hline Éclats assez longs $1 / 1,5$ & 3 & 13 & 10 & 2 & 3 & 31 \\
\hline Éclats longs $\quad 1 / 2$ & 2 & 1 & 2 & 1 & 3 & 9 \\
\hline Éclats laminaires $1 / 3$ & & 1 & & & & 1 \\
\hline
\end{tabular}

Tableau 3 : Le Raumarais, locus 1 : modules des enlèvements. Table 3: Le Raumarais, locus 1: types of flakes.

\begin{tabular}{|l|c|c|c|c|c|c|}
\hline Longueur & Surface & $\mathrm{C} / 1$ & $\mathrm{C} / 2$ & $\mathrm{C} / 2-3$ & $\mathrm{C} / 3$ & Total \\
\hline Éclats très petits $<2 \mathrm{~cm}$ & 7 & 16 & 16 & 9 & 4 & 52 \\
\hline Éclats petits 2 à $4 \mathrm{~cm}$ & 7 & 22 & 10 & 2 & 2 & 43 \\
\hline Éclats assez petits 4 à 6 cm & 1 & 6 & 6 & & 1 & 14 \\
\hline Éclats moyens 6 à $8 \mathrm{~cm}$ & & 2 & & & 1 & 3 \\
\hline
\end{tabular}

Tableau 4 : Le Raumarais, locus 1 : longueur des enlèvements. Table 4: Le Raumarais, locus 1: length of flakes.

- Les nucléus (fig. 4, $\left.\mathrm{n}^{\circ} 15,18,19\right)$ : Onze nucléus ont été récoltés au cours de la fouille; ce nombre relativement important peut s'expliquer par l'utilisation de petits galets et rognons desquels on ne pouvait tirer qu'un nombre réduit de lamelles, mais aussi par la proximité probable d'une aire de débitage. Le nucléus le plus gros pèse $122 \mathrm{~g}$, le plus petit $33 \mathrm{~g}$. Six sont réalisés sur rognons de silex partiellement roulés, deux sur rognons non roulés, deux sur galets.

- Les lames: On n'en compte que deux. La première est de même style que les lamelles : long. $6,1 \mathrm{~cm}$, larg. $2 \mathrm{~cm}$, ép. $0,6 \mathrm{~cm}$; la seconde, très épaisse, n'est guère utilisable.

- Les lamelles: Elles représentent $17 \%$ du matériel brut. Le style du débitage est celui de Coincy. Elles sont toutes de petite taille, leur longueur dépasse très rarement $4 \mathrm{~cm}$ et leur épaisseur se situe le plus souvent entre 2 et $3 \mathrm{~mm}$. Elles ont en majorité une largeur inférieure à $12 \mathrm{~mm}$. Les lamelles à deux pans dominent (52 lamelles, contre 15 à trois pans). Trois d'entre elles comportent des micro-retouches d'utilisation (tabl. 5 et 6 ). Les talons sont le plus souvent lisses ou filiformes (fig. 3), les bulbes sont peu développés, le bord du plan de frappe a fréquemment été préparé par enlèvement de petits éclats et d'esquilles. Une percussion directe au percuteur de pierre tendre est donc probable (Inizan $e t$ al. 1995).

Figure 3: Le Raumarais, locus 1 : typologie des talons (éclats entiers et fragments proximaux).

Figure 3: Le Raumarais, locus 1: typology of the platforms (from complete flakes and proximal fragments).

\begin{tabular}{|l|c|c|c|c|}
\hline Désignation & Surface & $\mathrm{C} / 1$ & $\mathrm{C} / 2$ & Total \\
\hline Lamelles entières & 7 & 15 & 12 & 34 \\
\hline Lamelles raccourcies & 1 & 7 & 6 & 14 \\
\hline Corps de lamelles & 1 & 3 & 1 & 5 \\
\hline Lamelles à bulbe enlevé & 2 & 4 & 1 & 7 \\
\hline Extr. proximales de lamelles & 1 & 4 & 1 & 6 \\
\hline Extr. distales de lamelles & 1 & 2 & 1 & 4 \\
\hline Débris de lamelles & & 1 & & 1 \\
\hline
\end{tabular}

Tableau 5: Le Raumarais, locus 1 : répartition des lamelles. Table 5: Le Raumarais, locus 1: distribution of small blades.

- Les microburins (tabl. 7; fig. 4, $\mathrm{n}^{\circ} 1$ à 5) : Ils sont peu nombreux, mais l'ensemble du matériel recueilli n'est pas suffisamment important pour juger de la fréquence d'utilisation du procédé de section oblique.

\section{Le matériel retouché}

Typologie et répartition sont résumées dans le tableau 8.

\section{Les éclats retouchés}

Ces éclats se scindent en deux catégories : les éclats denticulés et les éclats divers.

- Éclats denticulés: On note un fragment proximal d'éclat mince denticulé à retouches latérales directes semi abruptes et un éclat épais denticulé à partie proximale corticale comportant une série de retouches directes semi abruptes; cet éclat peut avoir été utilisé comme racloir.

- Éclats divers : Cinq éclats minces et un éclat épais entrent dans cette catégorie.

Parmi les éclats minces figurent trois éclats entiers à retouches directes latérales, dont deux à retouches semi abruptes, le troisième à retouches abruptes. On note également un éclat entier à retouches mixtes semi abruptes latérales et distales et un fragment proximal à retouches mixtes semi abruptes distales et latérales. L'éclat épais est à dos naturel cortical et comporte une série de retouches inverses semi abruptes interrompue par une cassure. On observe sur la face d'éclatement des zones lustrées à proximité de la retouche. 


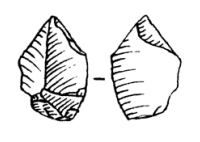

1

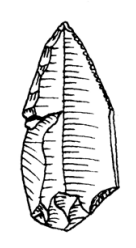

7

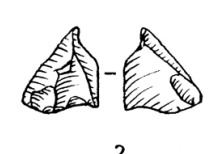

2

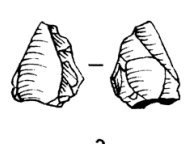

3

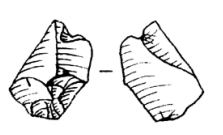

4
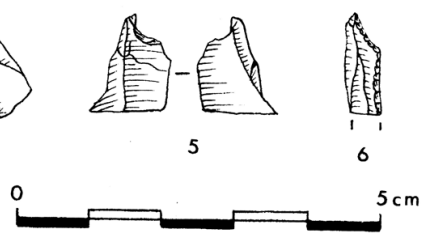

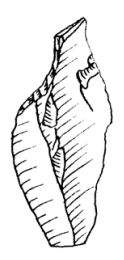

10

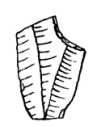

11

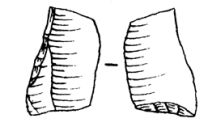

12

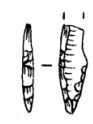

13

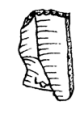

14

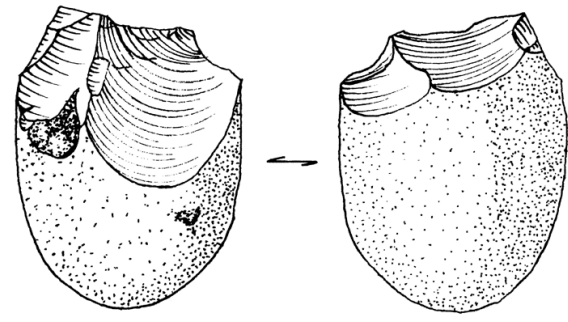

15

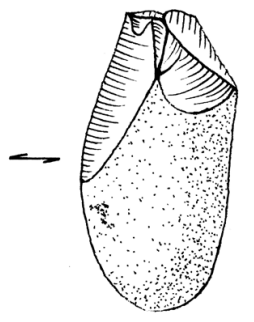

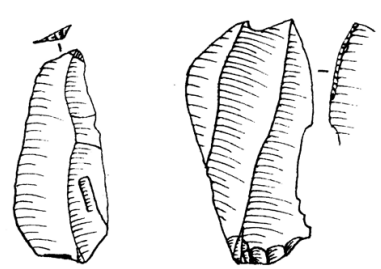

16

17

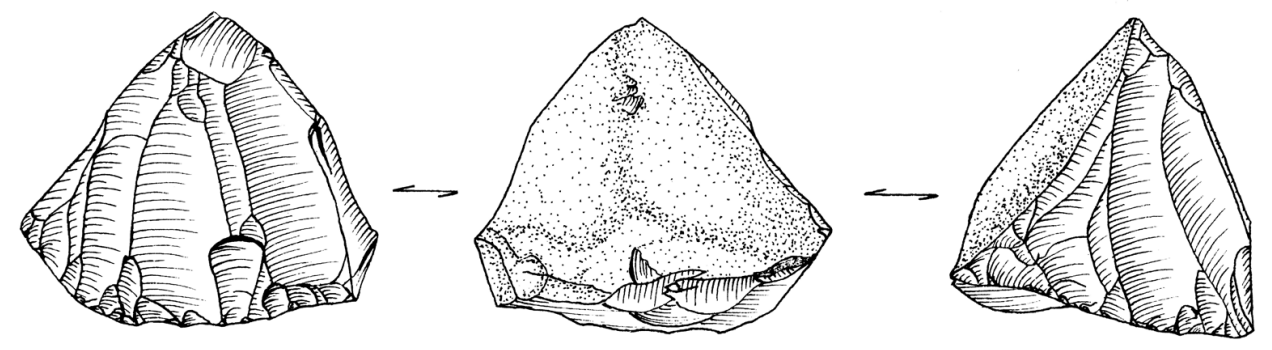

18
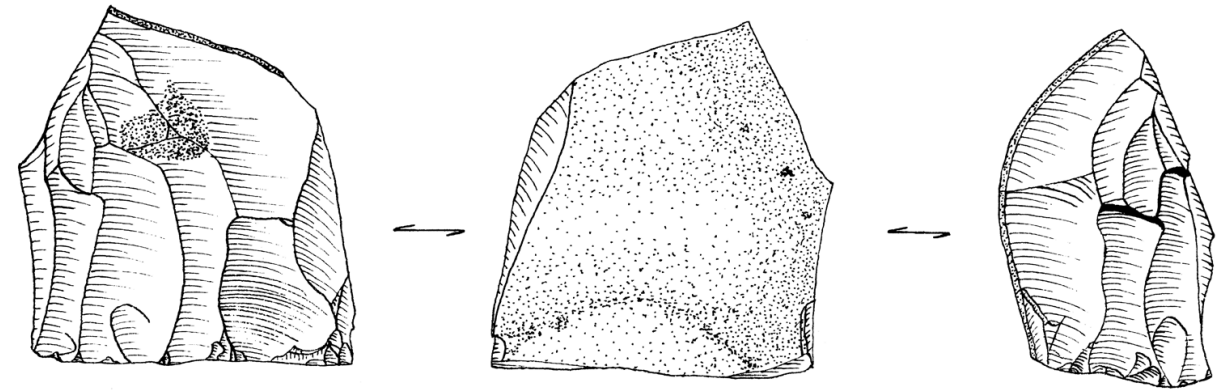

19

Figure 4 : Le Raumarais, locus $1: 1$ à 4, microburins proximaux; 5, microburin opposé à cassure; 6, : fragment de lamelle scalène; 7 , pointe à troncature oblique; 8, lamelle cassée à troncature oblique; 9, lamelle bordée; 10 et 11, lamelles cassées dans une coche; 12, fragment de pointe à base transversale; 13, fragment de pointe à retouche unilatérale; 14 et 16, lamelles à retouches distales; 17, lamelle à retouches partielles régulières; 15 et 19, nucléus unipolaires; 18 , nucléus pyramidal.

Figure 4: Locus 1: 1-4, proximal microburins; 5, microburin opposite to the break; fragment of scalene bladelet; 7, obliquely truncated point; 8, broken bladelet with oblique truncature; 9, bordered bladelet; 10-11, bladelets broken in a notch; 12, fragment of a transversal bottomed point; 13, fragment of a unilaterally retouched point; 14 and 16, distal retouched bladelets; 17, bladelet with partial regular retouches; 15 and 19, unipolar nuclei; 18, pyramidal nucleus. 


\begin{tabular}{|c|c|c|c|c|c|c|c|}
\hline \multirow[t]{2}{*}{ Désignation } & \multirow[t]{2}{*}{ Nombre } & \multirow{2}{*}{$\begin{array}{l}\text { Largeur } \\
<12 \mathrm{~mm}\end{array}$} & \multirow[t]{2}{*}{ Brûlées } & \multirow{2}{*}{$\begin{array}{l}\text { Avec } \\
\text { cortex }\end{array}$} & \multicolumn{3}{|c|}{ Nombre de pans } \\
\hline & & & & & 2 & 3 & 4 \\
\hline Lamelles entières & 34 & 23 & - & 3 & 24 & 7 & 3 \\
\hline Lamelles raccourcies & 14 & 10 & - & - & 11 & 3 & - \\
\hline Corps de lamelles & 5 & 4 & - & - & 4 & 1 & - \\
\hline Lamelles à bulbe enlevé & 7 & 5 & - & - & 5 & 2 & - \\
\hline Extrémités proximales de lamelles & 6 & 3 & - & - & 4 & 2 & - \\
\hline Extrémités distales de lamelles & 4 & 4 & 2 & - & 4 & - & - \\
\hline Débris de lamelles & 1 & 1 & - & - & - & - & 1 \\
\hline Total & 71 & 50 & 2 & 2 & 52 & 15 & 4 \\
\hline
\end{tabular}

Tableau 6 : Le Raumarais, locus 1 : description des lamelles. Table 6: Le Raumarais, locus 1: description of small blades.

\begin{tabular}{|c|c|c|c|c|c|}
\hline Type & $\mathrm{Nb}$ & $\begin{array}{c}\text { Coche } \\
\text { à droite }\end{array}$ & Dimensions & long. & Larg. \\
\hline $\begin{array}{c}\text { Microburins } \\
\text { proximaux }\end{array}$ & 4 & 4 & 0 à $1 \mathrm{~cm}$ & - & 4 \\
\hline $\begin{array}{c}\text { Microburins } \\
\text { opposés à cassure }\end{array}$ & 1 & 1 & 1 à $1,5 \mathrm{~cm}$ & 5 & 1 \\
\hline
\end{tabular}

Tableau 7 : Le Raumarais, locus 1 : description des microburins. Table 7: Le Raumarais, locus 1: description of microburins.

\begin{tabular}{|l|c|c|c|c|c|}
\hline \multicolumn{1}{|c|}{ Désignation } & Surface & $\mathrm{C} / 1$ & $\mathrm{C} / 2$ & $\mathrm{Nb}$ & Total partiel \\
\hline $\begin{array}{l}\text { Éclat mince } \\
\text { denticulé }\end{array}$ & & 1 & & 1 & \\
\hline Éclat épais denticulé & & & 1 & 1 & \\
\hline $\begin{array}{l}\text { Éclats minces } \\
\text { à retouches. } \\
\text { quelconques }\end{array}$ & 1 & 1 & 3 & 5 & \\
\hline $\begin{array}{l}\text { Éclats épais } \\
\text { à retouches } \\
\text { quelconques }\end{array}$ & & & 1 & 1 & 8 \\
\hline $\begin{array}{l}\text { Lamelle à retouches. } \\
\text { Partielles régulières }\end{array}$ & & 1 & 1 & 1 & \\
\hline Lamelle bordée & & 1 & 1 & 2 & \\
\hline $\begin{array}{l}\text { Lamelles cassées } \\
\text { dans une coche }\end{array}$ & & 1 & & & \\
\hline $\begin{array}{l}\text { Lamelles à retouches } \\
\text { distales }\end{array}$ & & 2 & 1 & 2 & \\
\hline $\begin{array}{l}\text { Lamelle cassée ̀̀ } \\
\text { troncature oblique }\end{array}$ & 1 & & & 1 & 7 \\
\hline $\begin{array}{l}\text { Pointe à troncature } \\
\text { très oblique }\end{array}$ & & & 1 & 1 & \\
\hline $\begin{array}{l}\text { Pointe à retouche } \\
\text { unilatérale }\end{array}$ & & 1 & 2 & 2 & 2 \\
\hline Lamelle scalène & & 1 & & 1 \\
\hline Microlithes divers & & & & \\
\hline Total général & & & & \\
\hline
\end{tabular}

Tableau 8 : Le Raumarais, locus 1 : inventaire du matériel retouché.

Table 8: Le Raumarais locus 1: distribution of retouched material.

\section{Lamelles, armatures et " divers »}

- Lamelles retouchées: Elles sont peu nombreuses. On distingue une lamelle à retouches partielles régulières (fig. 4, $\mathrm{n}^{\circ}$ 17), une lamelle bordée à retouches unilatérales (fig. 4, $\mathrm{n}^{\circ}$ 9), deux lamelles cassées dans une coche (fig. $4, \mathrm{n}^{\circ} 10$ et 11), deux lamelles à retouches distales abruptes (fig. 4, nº 4 ) et 16) et une lamelle cassée à troncature oblique exposée à gauche (fig. $4, \mathrm{n}^{\circ} 8$ ).

- Pointe à troncature oblique (fig. $4, \mathrm{n}^{\circ} 7$ ) : L'armature est réalisée sur la partie distale de la lamelle; l'angle de la pointe est de $45^{\circ}$, la troncature est exposée à gauche. On observe une fine retouche directe sur le bord opposé à la troncature.

- Pointe à retouche unilatérale (fig. 4, no 13) : Il s'agit d'un fragment d'armature étroite, très proche morphologiquement de la pointe de Sauveterre.

- Lamelle scalène (fig. $4, n^{\circ}$ 6) : On ne relève qu'un fragment distal à troncature légèrement concave.

- Microlithes divers (fig. 4, $\mathrm{n}^{\circ} 12$ ) : On note un petit éclat à retouches semi abruptes de la partie proximale pouvant provenir d'un outil, ainsi qu'un fragment de pointe à base transversale à retouches inverses $\left(n^{\circ} 12\right)$.

- Les "divers": Ils se résument à une plaquette de grès comportant à une de ses extrémités des traces de martelage (Longueur 11,4 cm, épaisseur $1 \mathrm{~cm}$ ).

\section{Le LOCUS 3}

Le gisement est situé en bordure nord du chemin rural allant du CD 403 au lieu dit "les landes de Digulleville», face à la station météorologique du Raumarais (point-origine du carroyage métrique en coordonnées Lambert I : x 296,445 - y 228,310). Le site fut repéré en juillet 1980 au cours d'une prospection ciblée sur les sondages réalisés par les géologues travaillant pour la COGEMA, où deux poches cendreuses accompagnées de pierres chauffées furent découvertes dans une coupe occasionnée par le creusement d'un caniveau en bordure du chemin. 
Après une série de sondages (fig. 5 ; tabl. 9), qui montrèrent que les vestiges archéologiques se raréfiaient proportionnellement à l'éloignement de la structure de combustion ainsi repérée, il fut décidé d'entreprendre la fouille sur une surface de $20 \mathrm{~m}^{2}$ centrée sur cette structure (Zone AE/1-4).

\section{Stratigraphie}

La fouille réalisée au-dessus du foyer présente d'une part un ensemble de couches archéologiques non remaniées et d'autre part une zone affectée par le creusement d'un fossé d'époque historique dont la date n'a pu être déterminée.

\section{Série stratigraphique se rapportant au fossé (fig. 6)}

- A : horizon végétal actuel.

- b : couche jaunâtre argileuse correspondant à la première phase de remplissage du fossé ; cette couche contient un certain nombre de vestiges lithiques, plus particulièrement au contact des parois du fossé.

- c : couche argileuse verdâtre; deuxième phase de remplissage du fossé contenant quelques silex taillés.

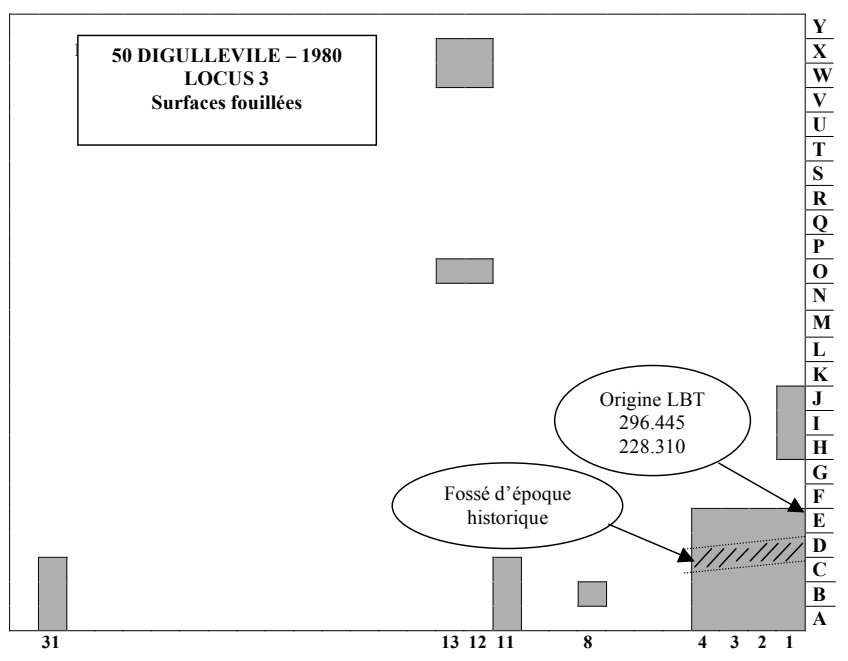

Figure 5 : Le Raumarais, locus 3 : plan des zones fouillées. Figure 5: Le Raumarais, locus 3: plan of the excavated areas.

\begin{tabular}{|c|c|c|}
\hline $\begin{array}{c}\text { Situation des } \\
\text { sondages }\end{array}$ & Surface en $\mathrm{m}^{2}$ & Nombre d'artefacts \\
\hline $\mathrm{B} / 8$ & 1 & 8 \\
\hline $\mathrm{A}, \mathrm{B}, \mathrm{C} / 11$ & 3 & 5 \\
\hline $\mathrm{A}, \mathrm{B}, \mathrm{C} / 31$ & 3 & 3 \\
\hline $\mathrm{A}, \mathrm{B}, \mathrm{C} / 42$ & 3 & 1 \\
\hline $\mathrm{O} / 12-13$ & 2 & 0 \\
\hline $\mathrm{W}, \mathrm{X} / 12-13$ & 4 & 1 \\
\hline
\end{tabular}

Tableau 9: Le Raumarais, locus 3 : situation des sondages. Table 9: Le Raumarais, locus 3: location of sondages.
- d : argile hydromorphe blanchâtre avec micro-encroûtement ferrugineux, vraisemblablement formée en présence d'eau stagnant ou circulant dans le fossé lors de sa période d'activité; celle-ci doit être ancienne puisqu'aucune trace de débris végétaux n’y est conservée.

- e : substrat; limon bigarré reposant sur un niveau de cailloutis, archéologiquement stérile.

Série stratigraphique se rapportant

à la zone non remaniée (fig. 7)

- A : horizon végétal actuel.

- a : couche brun noirâtre podzolisée, archéologiquement riche, correspondant à un ancien horizon végétal; le niveau d'accumulation du podzol se situe sur le paléosol enregistré " a/f »; il est possible que le transport des matières se soit trouvé bloqué et précipité au niveau «a/f » du fait d'une paléosurface.

- a/f : paléosurface mis en évidence par un niveau d'occupation caractérisé par une forte densité de vestiges lithiques.

- e : substrat; limon bigarré reposant sur un niveau de cailloutis, archéologiquement stérile.

- $\mathrm{f}$ : limon jaune bigarré correspondant à l'horizon d'accumulation de « $\mathrm{a}$ » homogène et compacté, peut être par l'occupation anthropique; le contact du sommet " $a / f$ » est franc (on peut noter que " $\mathrm{f}$ " est une couche d'épaisseur maximale de $15 \mathrm{~cm}$, centrée sur le foyer; on voit en effet dans la coupe qu'elle se termine en sifflet à environ $5 \mathrm{~m}$ de part et d'autre de celui-ci).

- $\mathrm{g}$ : couche cendreuse à gros fragments de charbon de bois occupant le fond de la cuvette du foyer.

- h : remplissage de la cuvette du foyer; cette couche semble être constituée de " $\mathrm{f}$ " rapportée afin de combler la cuvette; elle contient du matériel lithique chauffé, mais aussi indemne de toute trace de combustion.

\section{Structures}

Deux structures ont été reconnues au cours de la fouille : un fossé d'orientation W-SW/E-NE (fig. 6) et un foyer en cuvette (fig. 8 à 11).

Le fossé correspond sans doute à la levée de terre d'époque historique limitant la parcelle agricole. Cette levée de terre est lisible en coupe sous le talus actuel (datant des années 70). L'orientation du fossé n'est pas exactement parallèle au tracé actuel de la route. Son comblement s'est effectué en trois phases perceptibles sur la coupe (fig. 6) d, b, c. Le matériel lithique des couches b et c provient vraisemblablement des lambeaux de couche a et $\mathrm{f}$. Le profil du fossé est nettement dissymétrique, le versant le plus abrupt étant le versant sud, ce qui explique que le remplissage b soit déporté 


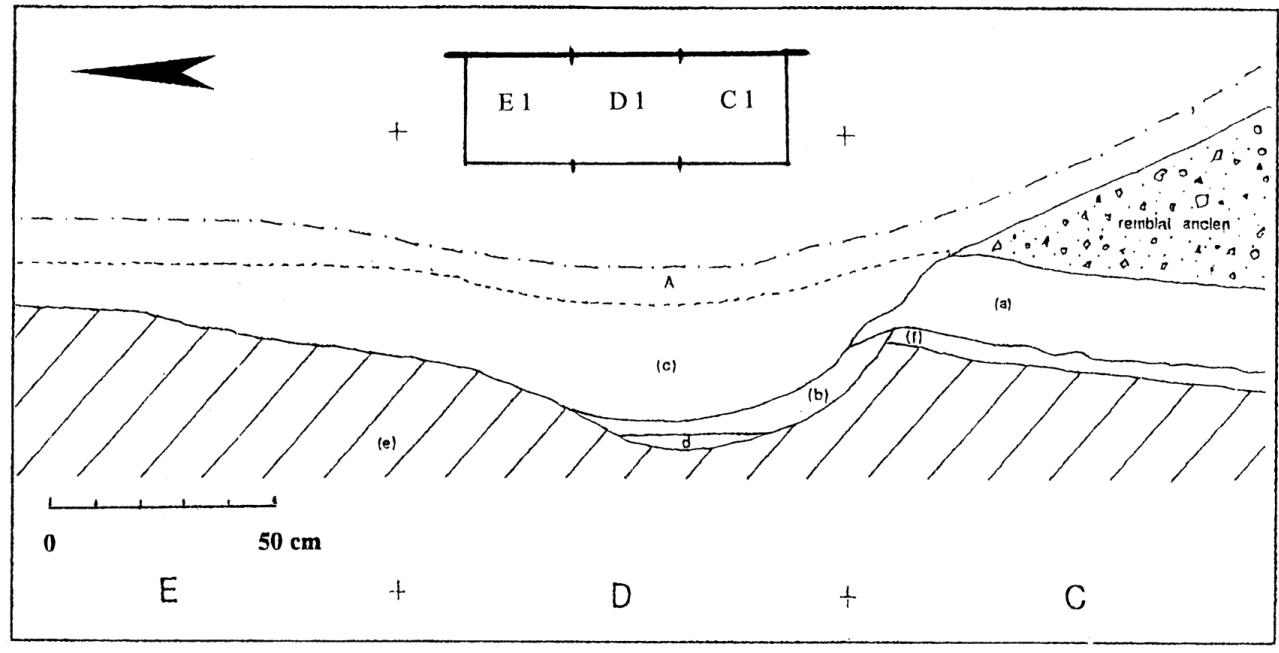

Figure 6 : Le Raumarais, locus 3 : coupe Nord-Sud du fossé.

Figure 6: Le Raumarais, locus 3 : North-South section of the ditch.

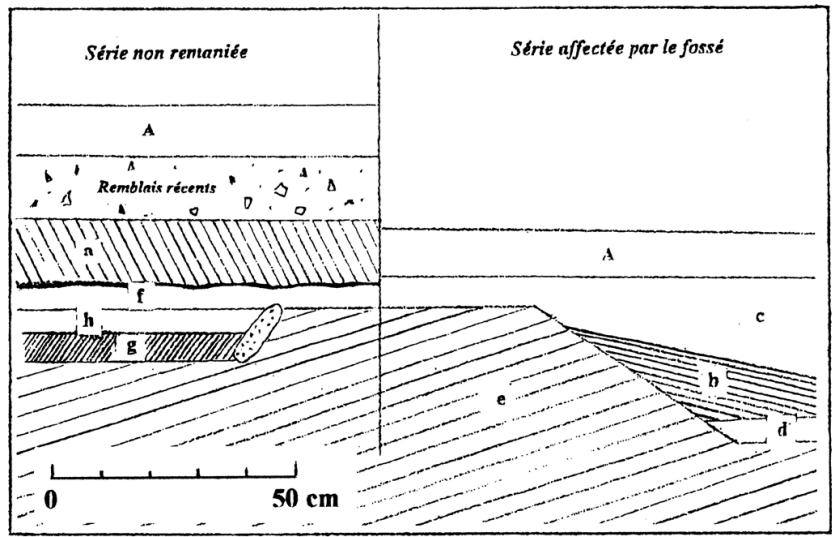

Figure 7 : Le Raumarais, locus 3 : coupe stratigraphique schématique.

Figure 7: Le Raumarais, locus 3: schematic stratigraphic section

« du côté sud, le plus éloigné du profil d'équilibre. La largeur est comprise entre 0,8 et $1 \mathrm{~m}$, la profondeur variant de 0,40 à $0,50 \mathrm{~m}$ à l'époque du creusement.

Le foyer est installé dans une cuvette creusée sous la couche mésolithique. La cuvette, d'une largeur de 1,20 m dans sa partie conservée, avait une profondeur d'environ $30 \mathrm{~cm}$, le fond était tapissé d'une couche charbonneuse recouverte par des blocs de grès chauffés englobés dans une couche cendreuse " $\mathrm{h}$ ». Il semble que la cuvette ait été comblée rapidement après l'utilisation du foyer. Quelques silex brûlés ont été rencontrés entre les blocs chauffés et au contact des parois de la cuvette. Le sommet du remplissage de la cuvette (couche $\mathrm{h}$ ) contient des éléments de débitage non chauffés.

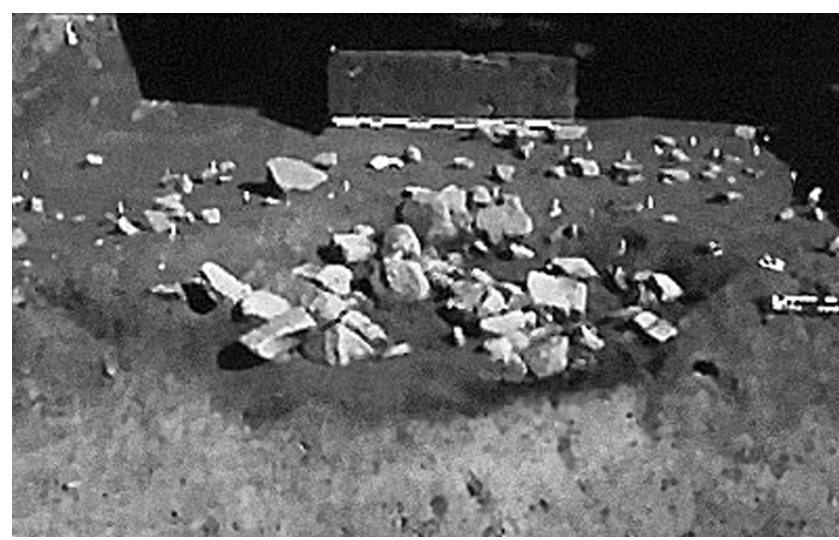

Figure 8 : Le Raumarais, locus 3 : vue du foyer.

Figure 8: Le Raumarais, locus 3: view of the hearth.

\section{Le matériel brut}

Typologie et répartition sont résumées dans le tableau 10 .

\section{Matériau utilisé}

Le matériau unique est également le silex. Comme sur le locus 1 , le silex gris clair a été le plus employé ( $72 \%$ contre $28 \%$ pour le silex gris foncé). L'examen des plages corticales conservées (sur 170 pièces) permet de relever quelques différences avec le locus 1. L'emploi de galets est plus fréquent, contrairement à celui de rognons non roulés; l'utilisation de rognons partiellement roulés est légèrement plus importante :

Sur le locus 1, les galets représentent $14 \%$, les rognons partiellement roulés $42 \%$, les rognons non roulés $44 \%$.

Sur le locus 3, les galets représentent $29 \%$, les rognons partiellement roulés $49 \%$, les rognons non roulés $22 \%$. 


\begin{tabular}{|c|c|c|c|c|c|c|c|c|c|c|c|}
\hline \multirow[b]{2}{*}{ Désignation } & \multicolumn{4}{|c|}{ Séquence affectée par le fossé } & \multicolumn{5}{|c|}{ Séquence non remaniée } & \multirow[b]{2}{*}{ Nombre } & \multirow{2}{*}{$\begin{array}{l}\text { Total } \\
\text { partie }\end{array}$} \\
\hline & $\begin{array}{c}\text { Surface } \\
\text { Divers }\end{array}$ & $\mathrm{b}$ & $\mathrm{b} / \mathrm{c}$ & $\mathrm{c}$ & cle & $\mathrm{a}$ & $\mathrm{a} / \mathrm{f}$ & $\mathrm{f}$ & $\mathrm{h}$ & & \\
\hline Nucléus unipolaires & 3 & & & & 2 & 1 & 2 & & & 8 & \\
\hline Nucléus prismatique angulaire & 1 & & & & & & & & & 1 & \\
\hline Nucléus à enlèvements croisés & & & & & & & & 3 & & 3 & \\
\hline Nucléus informes & 3 & & & 1 & & & & 2 & & 6 & 18 \\
\hline Éclats d'avivage & 5 & & & & & 1 & & 1 & & 7 & 7 \\
\hline Lames raccourcies & 2 & & & & & & & & & 2 & 2 \\
\hline Lamelles entières & 8 & & 3 & 3 & 4 & 7 & 6 & 3 & 2 & 36 & \\
\hline Lamelles raccourcies & 2 & 1 & & 3 & & 5 & & 3 & & 14 & \\
\hline Corps de lamelles & & & & 1 & & 4 & & & 1 & 6 & \\
\hline Lamelles à bulbe enlevé & 4 & & 1 & & & 1 & 1 & 1 & & 8 & 64 \\
\hline Extrémités prox. de lamelles & 5 & 2 & 1 & 3 & & 2 & 2 & & & 15 & \\
\hline Extrémités distales de lamelles & 1 & & & & & & & 1 & & 2 & \\
\hline Débris de lamelles & & & 1 & & & 2 & & & & 3 & 20 \\
\hline Microburins & & & & 1 & & 1 & & & & 2 & 2 \\
\hline Éclats non utilisés & 127 & 9 & 25 & 59 & 15 & 132 & 43 & 79 & 28 & 517 & \\
\hline Esquilles $(<1 \mathrm{~cm})$ & 14 & 1 & 3 & 16 & 5 & 73 & 15 & 22 & 3 & 152 & \\
\hline Cassons & 22 & 2 & 2 & 10 & 1 & 35 & 8 & 20 & 2 & 102 & \\
\hline Total général & & & & & & & & & & & 884 \\
\hline
\end{tabular}

Tableau 10 : «Le Raumarais » locus 3 : inventaire du matériel brut. Table 10: "Le Raumarais" locus 3: distribution of unworked material.

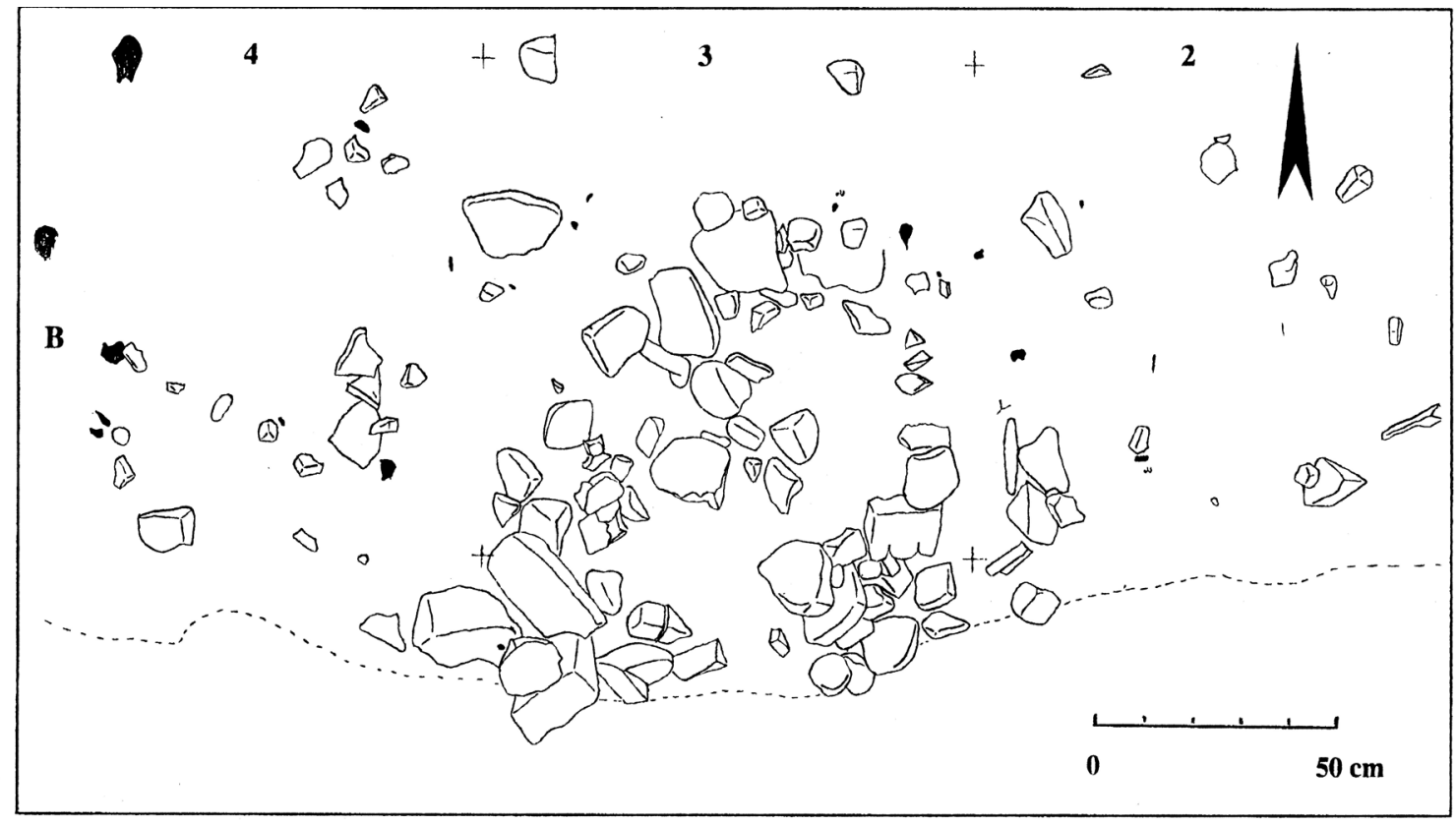

Figure 9 : Le Raumarais, locus 3 : plan du foyer.

Figure 9: Le Raumarais, locus 3: plan of the hearth.

\section{Produits de débitage}

Les produits de débitage sont également comparables (tabl. 11), le groupe le plus important (87\%) étant constitué d'éclats non utilisés, de cassons et d'esquilles. Les lamelles sont peu nombreuses $(9,5 \%)$, mais elles restent le support de base de l'outillage. Les lames ainsi que les microburins sont très rares.

\section{Module des enlèvements}

Les calculs ont été effectués à partir des enlèvements entiers. Un groupe représentant $95 \%$ du débitage se déta- 


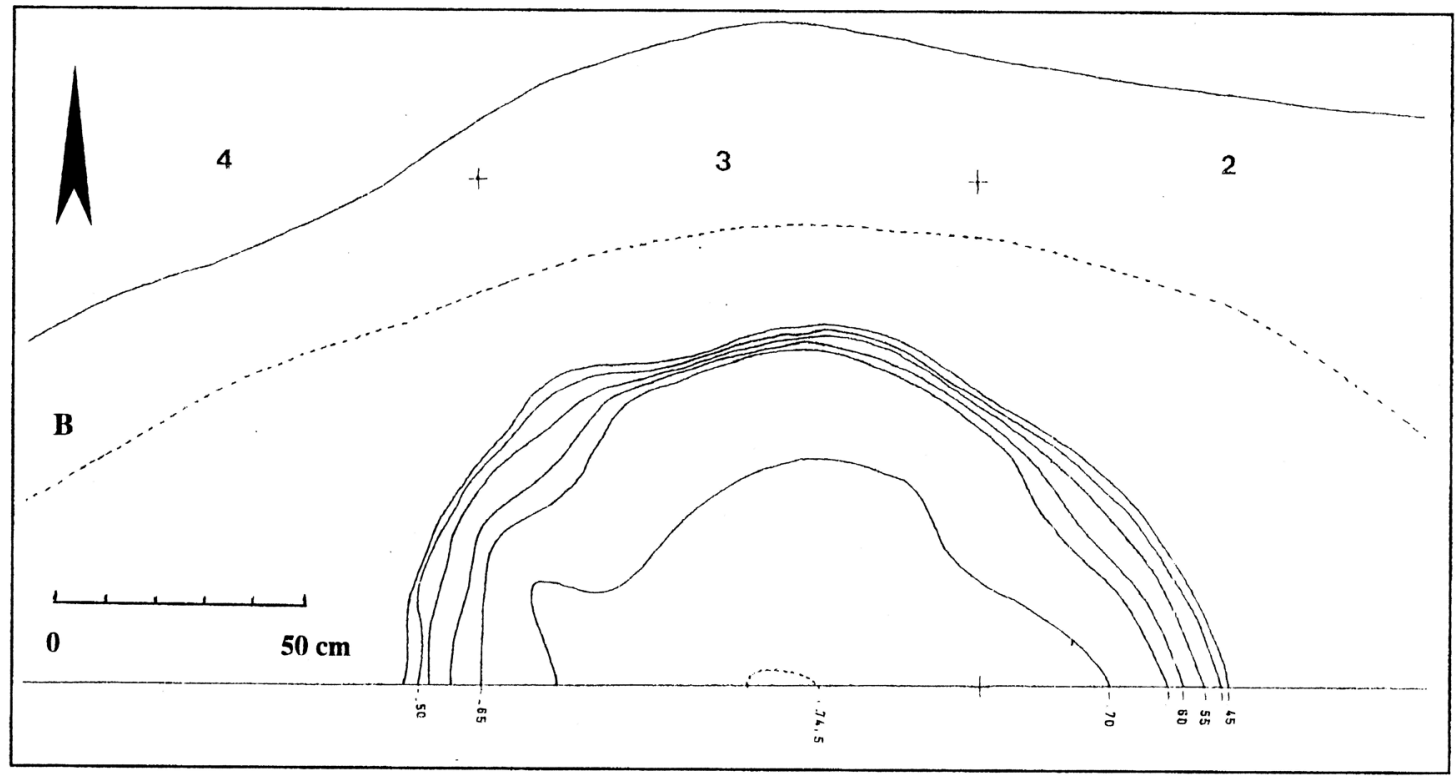

Figure 10 : Le Raumarais, locus 3 : relevé topographique de la cuvette du foyer. (équidistance des courbes de niveau : $5 \mathrm{~cm})$.

Figure 10: Le Raumarais, locus 3: Topographical survey of the hearth hollow (interval of contours: $5 \mathrm{~cm}$ ).

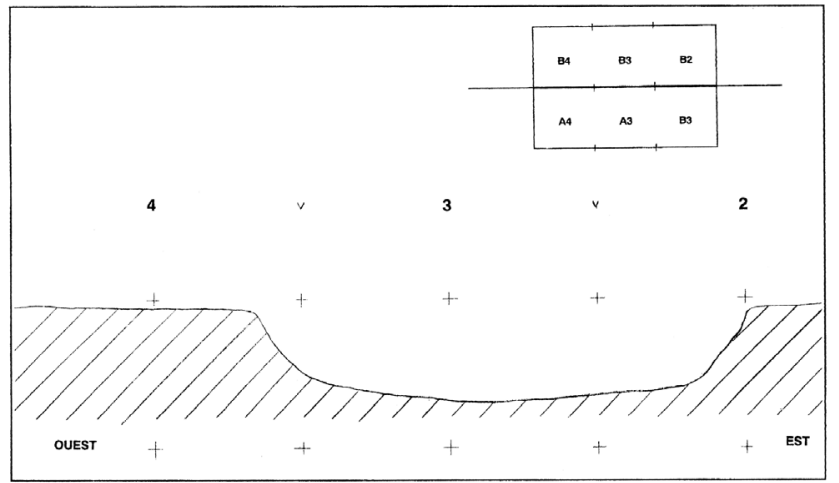

Figure 11 : Le Raumarais, locus 3 : coupe est-ouest du foyer. Figure 11: Le Raumarais, locus 3: E-W section of the hearth.

\begin{tabular}{|l|c|c|c|}
\hline Désignation & Nombre & Poids & dont brûlés \\
\hline Éclats entiers & 264 & $1576 \mathrm{~g}$ & 21 \\
\hline Éclats proximaux & 119 & 295 & 21 \\
\hline Éclats mésiaux & 46 & 60 & 18 \\
\hline Éclats distaux & 88 & 219 & 17 \\
\hline Cassons & 102 & 293 & 60 \\
\hline Esquilles $<1 \mathrm{~cm}$ & 152 & 47 & 39 \\
\hline
\end{tabular}

Tableau 11 : Le Raumarais, locus 3 : répartition des éclats. Table 11: Le Raumarais, locus 3: distribution of flakes.

che nettement; il est formé d'éclats larges, d'éclats très larges et d'éclats assez longs (tabl. 12 et 13). Les enlèvements sont dans l'ensemble assez petits : $86 \%$ des éclats ne dépassent pas $4 \mathrm{~cm}$.
Les modules principaux sont les suivants : éclats très larges $(\mathrm{L} / \mathrm{l}>1)$ et très petits $(<2 \mathrm{~cm}): 19 \%$; éclats larges $(1 / 1)$ et petits $(2$ à $4 \mathrm{~cm}): 16 \%$; éclats larges (1/1) et très petits $(<2 \mathrm{~cm}): 16 \%$; éclats assez longs $(1 / 1,5)$ et petits (2 à $4 \mathrm{~cm}): 14 \%$.

On note une prédominance des talons lisses (53\%); les autres types (corticaux, punctiformes, filiformes) se rencontrent dans une fourchette de 8 à un peu plus de $11 \%$ (fig. 12).

\section{Les nucléus}

Ils sont tous à éclats; sept d'entre eux comportent des enlèvements lamellaires; seize sont en silex gris clair, deux en silex gris foncé, ce qui concorde parfaitement avec l'ensemble du débitage. Le plus petit pèse $18 \mathrm{~g}$, le plus lourd $399 \mathrm{~g}$; si l'on excepte ce dernier, le poids moyen s'établit aux alentours de $57 \mathrm{~g}$.

\section{Les lames et lamelles}

Seules deux lames raccourcies ont été trouvées.

Le pourcentage des lamelles (cf. tabl. 14) est faible dans le locus $3(9,5 \%)$, comparé au locus 1 où il atteint $17,40 \%$; leur style est celui de Coincy, la majorité d'entre elles ont une largeur inférieure à $12 \mathrm{~mm}$, les lamelles à deux pans dominent. Leurs talons se répartissent de la façon suivante : lisses 26, punctiformes 8, filiformes 19, indéterminables 12 . 


\begin{tabular}{|ll|c|c|c|c|c|c|c|c|c|c|}
\hline Modules & & Surface & $\mathrm{b}$ & $\mathrm{b} / \mathrm{c}$ & $\mathrm{c}$ & $\mathrm{c} / \mathrm{e}$ & $\mathrm{a}$ & $\mathrm{a} / \mathrm{f}$ & $\mathrm{f}$ & $\mathrm{h}$ & Total \\
\hline Très larges & $>1$ & 17 & 1 & 4 & 12 & 1 & 18 & 3 & 13 & 7 & 76 \\
\hline Larges & $1 / 1$ & 21 & 3 & 3 & 6 & 5 & 28 & 14 & 17 & 3 & 100 \\
\hline Assez longs & $1 / 1,5$ & 19 & 1 & 6 & 9 & 2 & 22 & 6 & 7 & 2 & 74 \\
\hline Longs & $1 / 2$ & 3 & - & - & 1 & - & 3 & 1 & 4 & 1 & 13 \\
\hline Laminaires & $1 / 3$ & - & - & - & 1 & - & - & - & - & - & 1 \\
\hline
\end{tabular}

Tableau 12 : Le Raumarais, locus 3 : répartition des différents modules.

Table 12: Le Raumarais, locus 3: types of flakes.

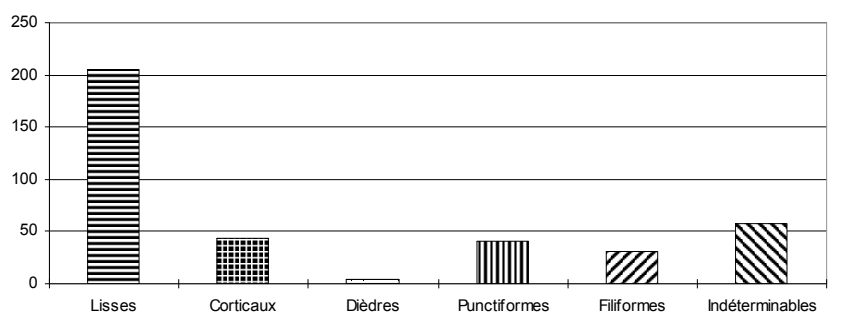

Figure 12 : Le Raumarais, locus 3 : typologie des talons (éclats entiers et fragments proximaux).

Figure 12: Le Raumarais, locus 3: typology of platforms (complete flakes and proximal fragments).

\section{Les microburins}

Seuls deux exemplaires ont été récoltés au cours de la fouille; il s'agit de microburins proximaux à coche à droite. La technique de section oblique semble peu utilisée, ce qui n'est pas étonnant sur un site à forte proportion de lamelles à dos; la fracturation des lamelles par flexion était probablement pratiquée.

\section{Le matériel retouché}

Caractéristiques et répartition sont résumées dans le tableau 15.

\section{Outils du fond commun}

Les grattoirs

Au nombre de 15, ils représentent $22 \%$ du matériel retouché, ce qui est important comparativement au Tardenoisien et au Groupe Breton où ils sont dans l'ensemble peu nombreux. Contrairement à ce qui a été écrit (Chancerel et Locard, 1991, Ghesquière et al., 2000), leur importance n'est pas liée à la proximité du site campaniforme (locus 2) (Letterlé et Verron, 1986), où des grattoirs de mêmes types étaient présents en surabondance, car 11 des 15 grattoirs ont été trouvés concentrés sur $6 \mathrm{~m}^{2}$ dans la zone où se situait le foyer (rappelons que le locus 2 était éloigné de plus de $450 \mathrm{~m}$ - cf. fig. 2).

Les grattoirs se rencontrent dans des proportions semblables dans le Limbourgien moyen : 20,6\% à Oirshot

\begin{tabular}{|l|c|c|c|c|c|c|c|c|c|c|}
\hline Longueur & Surface & $\mathrm{b}$ & $\mathrm{b} / \mathrm{c}$ & $\mathrm{c}$ & $\mathrm{c} / \mathrm{e}$ & $\mathrm{a}$ & $\mathrm{a} / \mathrm{f}$ & $\mathrm{f}$ & $\mathrm{h}$ & Total \\
\hline Très petits $<2 \mathrm{~cm}$ & 23 & 2 & 2 & 15 & 2 & 47 & 8 & 16 & 7 & 123 \\
\hline Petits 2 à 4 cm & 22 & 3 & 10 & 9 & 4 & 22 & 12 & 16 & 5 & 104 \\
\hline Assez petits 4 à 6 cm & 11 & - & 1 & 5 & 2 & 2 & 3 & 8 & 1 & 33 \\
\hline Moyens 6 à 8 cm & 2 & - & - & - & - & - & - & 1 & - & 3 \\
\hline Assez grands 8 à 10 cm & - & - & - & - & - & - & 1 & - & - & 1 \\
\hline
\end{tabular}

Tableau 13 : Le Raumarais, locus 3 : longueur des enlèvements.

Table 13: Le Raumarais, locus 3: length of flakes.

\begin{tabular}{|l|c|c|c|c|c|c|}
\hline \multirow{2}{*}{ Désignation } & \multirow{2}{*}{ Nombre } & \multirow{2}{*}{$\begin{array}{c}\text { Largeur } \\
\text { Lamelles entières }\end{array}$} & \multirow{2}{*}{ brûlées } & \multirow{2}{*}{$\begin{array}{c}\text { Avec } \\
\text { cortex }\end{array}$} & \multicolumn{2}{|c|}{ Nombre de pans } \\
\cline { 5 - 8 } & & 16 & 1 & 4 & 2 & 3 \\
\hline Lamelles raccourcies & 14 & 9 & 1 & - & 11 & 16 \\
\hline Corps de lamelles & 6 & 6 & - & - & 6 & - \\
\hline Lamelles à bulbe enlevé & 8 & 7 & - & 1 & 6 & 2 \\
\hline Extr. proximales de lamelles & 15 & 6 & 1 & - & 10 & 5 \\
\hline Extr. distales de lamelles & 2 & 2 & - & - & 2 & - \\
\hline Débris de lamelles & 3 & 1 & - & - & 2 & 1 \\
\hline Total & 84 & 47 & 3 & 5 & 57 & 27 \\
\hline
\end{tabular}

Tableau 14 : Le Raumarais, locus 3 : description des lamelles. Table 14: Le Raumarais, locus 3: description of small blades. 


\begin{tabular}{|c|c|c|c|c|c|c|c|c|c|}
\hline \multirow[b]{2}{*}{ Désignation } & \multicolumn{3}{|c|}{ Séquence affectée par le fossé } & \multicolumn{4}{|c|}{ Séquence non remaniée } & \multirow[b]{2}{*}{$\mathrm{Nb}$} & \multirow{2}{*}{$\begin{array}{c}\text { Total } \\
\text { partiel }\end{array}$} \\
\hline & $\begin{array}{l}\text { Surface } \\
\text { Divers. }\end{array}$ & c & cle & a & $\mathrm{a} / \mathrm{f}$ & $\mathrm{f}$ & $\mathrm{h}$ & & \\
\hline Grattoirs simples sur éclats & 1 & 1 & & 1 & 1 & 1 & & 5 & \\
\hline Grattoirs à front débordant & 4 & 1 & & 1 & & & & 6 & \\
\hline Grattoir ovalaire & & & 1 & & & & & 1 & \\
\hline Grattoirs ogivaux & & & & 1 & 1 & & & 2 & 14 \\
\hline Grattoir denticulé & & & & & & 1 & & 1 & \\
\hline Éclats épais à retouches quelconques & 1 & & 1 & 2 & 1 & & & 5 & \\
\hline Éclats minces à retouches quelconques & 2 & 2 & & 2 & & & 2 & 8 & \\
\hline Racloirs & 1 & & & 1 & & & & 2 & 16 \\
\hline Perçoir & 1 & & & & & & & 1 & 1 \\
\hline Lame à troncature oblique & & & & 1 & & & & 1 & \\
\hline Lamelles épaisses à retouches régulières & 2 & & & & 1 & & & 3 & \\
\hline Couteau à dos & 1 & & & & & & & 1 & 5 \\
\hline Lamelle à bord abattu atypique & & & & 1 & & 1 & & 2 & \\
\hline Lamelle à retouches partielles régulières & & & & & & 1 & & 1 & \\
\hline Lamelle à retouches continues & & 1 & & & & & & 1 & \\
\hline Lamelles bordées & & 1 & & & 1 & & & 2 & \\
\hline Lamelle cassée au dessus d'une coche & & & & 1 & & & & 1 & \\
\hline Lamelles cassées dans une coche & & & & 1 & 1 & & & 2 & \\
\hline Lamelles à troncature oblique & 1 & & & 2 & & & & 3 & 12 \\
\hline Pointe à retouche unilatérale & & & & 1 & & & & 1 & \\
\hline Pointes à bord abattu & 1 & & & 1 & & & & 2 & 3 \\
\hline Fragts. de lamelles étroites à bord abattu & & & 2 & 8 & 3 & 1 & 1 & 15 & \\
\hline Lamelles étroites à bord abattu tronquées & 1 & & & 1 & & & & 2 & 17 \\
\hline Pointe de Tardenois à base concave & & & & 1 & & & & 1 & 1 \\
\hline Total général & & & & & & & & & 69 \\
\hline Débris de microlithes & & & & & & & & 7 & \\
\hline Débris de lamelles retouchées & & & & & & & & 5 & \\
\hline
\end{tabular}

Tableau 15 : Le Raumarais, locus 3 : inventaire du matériel retouché. Table 15: Le Raumarais, locus 3: distribution of retouched material.

V - GQW ; 25,9 \% à Oirschot V - Silex (Rozoy, 1978). Dans la Somme, ils peuvent être très abondants dans des séries diachrones (Lihus II, Thennes IV, Hangest II. 3) et présenter des taux très différents dans des gisements sensiblement contemporains : Lihus II, Conty, Hangest II nord (Ducrocq, 2001). Les nombreux grattoirs trouvés sur le locus 3 des Raumarais peuvent être liés à une activité spécifique exercée sur le site. Ils sont tous réalisés sur éclats, généralement de petite taille. Huit d'entre eux proviennent de galets; on en distingue cinq types.

- Grattoirs simples sur éclat (fig. 13, $\mathrm{n}^{\circ} 1,2,3$ ). Trois sont tirés de galets, deux conservent une plage de cortex à l'emplacement du talon, ce qui devait en faciliter la préhension; ils sont de petite taille mais robustes. Mis à part le $\mathrm{n}^{\circ} 1$, leur longueur ne dépasse pas $2 \mathrm{~cm}$, leur épaisseur moyenne est de $8 \mathrm{~mm}$. Le $\mathrm{n}^{\circ} 3$ comporte à la base une amorce de retouches, toutefois insuffisante pour le qualifier de grattoir double.

- Grattoirs à front débordant (fig. 13, $\mathrm{n}^{\circ} 4,5,8,9,12$ ). Ce sont les plus nombreux; quatre sont tirés de galets, un autre provient d'un rognon partiellement roulé, le $\mathrm{n}^{\circ} 9$ est réalisé sur un éclat dont le cortex a été éliminé. Sur quatre d'entre eux, le prolongement du front ne suit pas l'arrondi de la partie distale; celui-ci est réalisé par une ligne de retouches droites des deux bords, qui forment un angle plus ou moins net avec la partie distale. Les retouches constituant le prolongement du front sont légèrement plus abruptes et étaient peut-être destinées essentiellement à une meilleure prise de l'outil.

- Grattoir ovalaire (fig. 13, $\mathrm{n}^{\circ} 14$ ). Le seul exemplaire recueilli semble avoir été beaucoup utilisé à en juger du fort esquillement des bords. Sa forme initiale devait être différente. La forme ovalaire actuelle n'est probablement que le résultat d'une usure.

- Grattoirs ogivaux (fig. 13, n 6 et 7). Ils sont réalisés sur petits éclats; leur épaisseur ne dépasse pas $6 \mathrm{~mm}$. Leurs bords retouchés en arc de cercle se rejoignent dans l'axe de la pièce et forment un angle aigu. Ce type ne semble pas courant dans l'Épipaléolithique. 


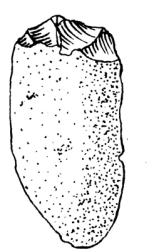

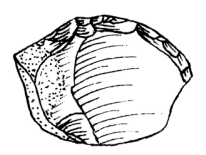

2

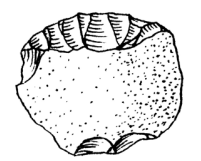

3

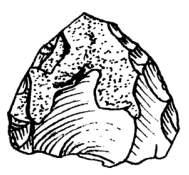

6

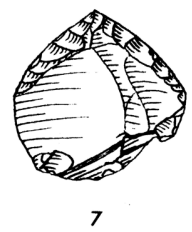

12

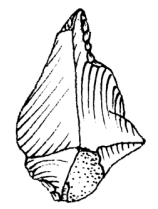

17

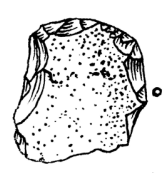

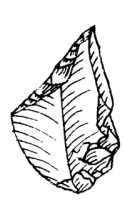

18

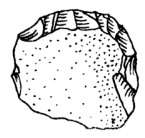

8

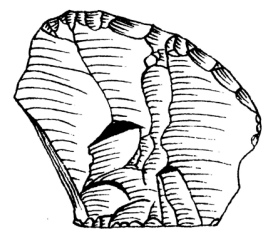

13
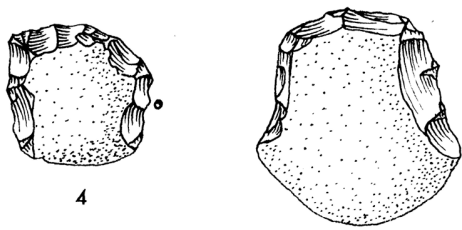

5
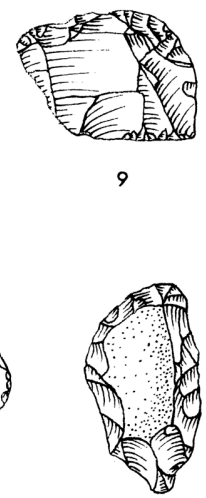

14

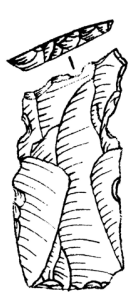

19

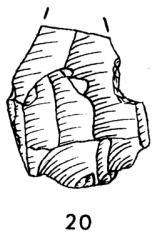

20
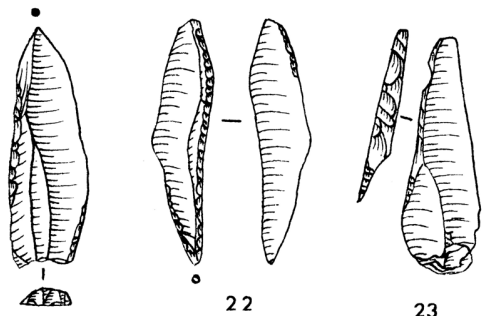

23
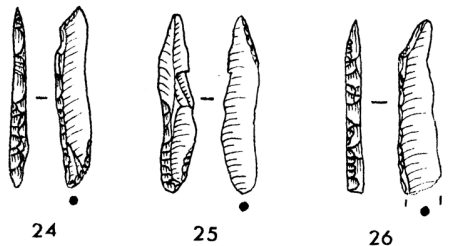

21

0

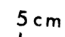

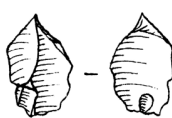

28

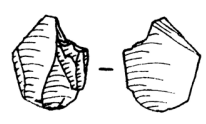

29

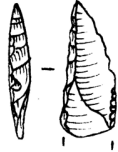

30

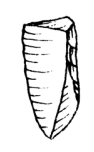

31

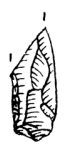

32

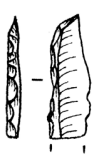

33
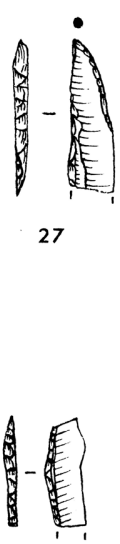

34

Figure 13 : «Le Raumarais » locus $3: 1$ à 3, grattoirs simples sur éclat; 4, 5, 8, 9, 12, grattoirs à front débordant; 6, 7, grattoirs ogivaux; 10 : couteau à dos; $11:$ grattoir denticulé; $13,15:$ racloirs; $14:$ grattoir ovalaire; $16:$ lame à troncature oblique; $17:$ perçoir; 18,19 , 23 : lamelles à troncature oblique; 20 : lamelle cassée au-dessus d'une coche; 21 : pointe du Tardenois à base concave; $22,25:$ pointes à bord abattu; 26, 33, 34 : fragments de lamelles étroites à bord abattu; 27 : fragment de pointe à retouche unilatérale; 30 : débris de microlithe; 31 : lamelle à retouches partielles régulières; 32 : lamelle à retouches continues.

Figure 13: "Le Raumarais" locus 3: 1-3, simple scapers on flakes; 4, 5, 8, 9, 12, beaked scapers; 6-7, ogival scapers; 10, backed knives; 11, denticulate scaper; 13, 15, side scapers; 14, oval scaper; 16, obliquely truncated blade; 17, borer; 18, 19, 23, obliquely truncated bladelets; 20, bladelet broken above a notch; 21, Tardenois point with concave base; 22, 25, edged points; 26, 33, 34, fragments of narrow edged bladlets; 27 , fragment of unilateral retouched point; 30, microlithic debris; 31 bladelet with partial even retouch; 32, bladelet with continuous retouch. 
- Grattoir denticulé (fig. 13, n ${ }^{\circ} 11$ ). La denticulation en est réalisée par une série de retouches contigues de type clactonien.

Les autres pièces

- Éclats retouchés. Ces éclats se répartissent en neuf éclats entiers, un fragment proximal et trois fragments distaux. On note huit éclats minces et cinq éclats épais à retouches quelconques (tabl. 16).

- Racloirs (fig. 13, n ${ }^{\circ} 13$ et 15). On distingue un racloir convexe $\left(n^{\circ} 13\right)$ et un racloir concave $\left(n^{\circ} 15\right)$, à retouches directes.

- Perçoir (fig. $13, \mathrm{n}^{\circ} 17$ ). Il est réalisé sur éclat et sa pointe est dégagée par retouches bilatérales directes; il entre dans la catégorie des perçoirs fins.

- Lame à troncature oblique. Le $n^{\circ} 6$, fig. 13 est une section de lame comportant une troncature oblique exposée à gauche, opposée à une série de retouches continues semi-abruptes, parallèle à la troncature dont l'obliquité est de $15^{\circ}$.

- Lamelles épaisses à retouches régulières. Leur épaisseur est supérieure à $4 \mathrm{~mm}$; on note deux lamelles entières à retouches partielles inverses unilatérales et une extrémité distale à retouches partielles inverses bilatérales.

- Couteau à dos (fig. 13, n 10). Il est de petite taille (long. $4,5 \mathrm{~cm})$; le dos est façonné par retouches abruptes irrégulières.

- Lamelles à bord abattu atypique. Elles sont représentées par deux fragments distaux, dont un à retouches bilatérales.

- Lamelle à retouches partielles régulières (fig. 13, $\mathrm{n}^{\circ} 31$ ). Une extrémité distale de lamelle à retouches partielles régulières abruptes d'un bord résulte peut-être de la confection d'une lamelle à bord abattu.

- Lamelle à retouches continues (fig. $13, \mathrm{n}^{\circ} 32$ ). Une lamelle raccourcie porte des retouches continues unilatérales.
- Lamelles bordées. On trouve une lamelle entière et une lamelle raccourcie à micro-retouches unilatérales (bordage).

- Lamelle cassée au-dessus d'une coche (fig. 13, $\mathrm{n}^{\circ} 20$ ). Cette extrémité proximale de lamelle est cassée au-dessus d'une coche située à droite.

- Lamelles cassées dans une coche (fig. 13, $n^{\circ} 29$ ). On note une extrémité distale de lamelle résultant probablement de la confection d'une lamelle à bord abattu, ainsi qu'une extrémité proximale dont la cassure a été réalisée par flexion.

- Lamelles à troncature oblique (fig. 13, $\mathrm{n}^{\circ} 18,19,23$ ). Trois lamelles montrent des troncatures exposées à gauche. L'obliquité des troncatures pour deux d'entre elles (18 et 19) est de $30^{\circ}$. La lamelle ${ }^{\circ} 23$, malgré une obliquité de sa troncature de $70^{\circ}$, n'a pas été considérée comme une pointe à base naturelle en raison de son aspect général et notamment de la retouche de son extrémité distale.

\section{Armatures}

- Pointes à bord abattu (fig. 13, n 22 et 25). Les deux exemplaires ont un dos gibbeux. La pointe $\mathrm{n}^{\circ} 25$, réalisée sur lamelle étroite, a son bord abattu partiellement. L'extrémité distale de chacune comporte une retouche inverse opposée au bord retouché. Ces pointes sont proches morphologiquement des pointes de Sauveterre à retouches partielles, mais elles n'ont pas le caractère effilé de ces dernières. Il peut s'agir d'un type régional. Dans les graphiques cumulatifs nous les avons associées aux pointes à retouche unilatérale $\left(\mathrm{n}^{\circ} 51 \mathrm{de}\right.$ la liste typologique de J.-G. Rozoy).

- Fragment de pointe à retouche unilatérale (fig. 13, $\mathrm{n}^{\circ} 27$ ). La pointe est réalisée sur la partie proximale d'une lamelle étroite. Le bord abattu est rectiligne, le bord opposé est retouché partiellement pour former la pointe.

- Fragments de lamelles étroites à bord abattu (fig. 13, $\mathrm{n}^{\circ} 26$, 33, 34). 15 fragments ont été recueillis, parmi lesquels on

\begin{tabular}{|c|c|c|c|c|c|}
\hline \multicolumn{2}{|c|}{ Retouche } & \multicolumn{2}{|c|}{ Éclats entiers } & \multirow{2}{*}{$\begin{array}{l}\text { Fragments proximaux } \\
\text { minces }\end{array}$} & \multirow{2}{*}{$\begin{array}{c}\text { Fragments distaux } \\
\text { minces }\end{array}$} \\
\hline & & minces & épais & & \\
\hline \multirow[t]{3}{*}{ Mode } & Abrupte & 1 & & & 1 \\
\hline & Semi-abrupte & 2 & 5 & 1 & 1 \\
\hline & Plate & 1 & & & 1 \\
\hline \multirow[t]{2}{*}{ Ampleur } & Marginale & 1 & 3 & 1 & 2 \\
\hline & Profonde & 3 & 2 & & 1 \\
\hline \multirow[t]{2}{*}{ Orientation } & Directe & 3 & 3 & & 3 \\
\hline & Inverse & 1 & 2 & 1 & \\
\hline \multirow[t]{2}{*}{ Disposition } & Distale & 1 & 2 & & 2 \\
\hline & Latérale & 3 & 3 & 1 & 1 \\
\hline \multirow[t]{3}{*}{ Régularité } & Régulière & 3 & 1 & & 1 \\
\hline & Irrégulière & 1 & 4 & 1 & 2 \\
\hline & Total & 4 & 5 & 1 & 3 \\
\hline
\end{tabular}

Tableau 16 : Le Raumarais, locus 3 : description des éclats retouchés. Table 16: Le Raumarais, locus 3: description of retouched flakes. 
note quatre extrémités distales dont une avec retouches du deuxième bord, sept corps de lamelles dont trois ont un piquant trièdre conservé, et quatre extrémités proximales.

- Lamelles étroites à bord abattu tronquées. On a un fragment et une lamelle entière (fig. $13, n^{\circ} 24$ ), probablement montée en pointe. L'obliquité de la troncature est de $52^{\circ}$, la partie proximale comporte une retouche opposée au bord abattu.

- Pointe du Tardenois à base concave (fig. 13, n²1). C'est une pointe à retouches partielles d'un bord et piquant trièdre conservé. La retouche de la base est directe et abrupte.

- Débris de microlithes (fig. 13, n 30). La série comporte sept débris de microlithes non identifiables, dont deux à retouches abruptes, ainsi qu'un piquant trièdre ayant donné un microburin coche à droite. Le $\mathrm{n}^{\circ} 30 \mathrm{a}$ pu être été utilisé en l'état comme armature; sa base comporte un enlèvement transversal peut-être volontaire.

\section{Divers}

On y relève les éléments suivants :

- Une plaquette lissée en schiste, de forme rectangulaire (L. 9,6 cm, 1. 3,2 cm, ép. $1 \mathrm{~cm}$ ) ; le lissage affecte une seule face de la plaquette ainsi que ses deux bords.

- Un fragment de grès comportant une extrémité martelée (retouchoir?).

- Un fragment de grès quartzite à surface entièrement lissée, qui semble avoir été utilisé comme enclume; on peut observer sur trois côtés une concentration de traces d'impacts.

- Un tesson de céramique pré ou protohistorique à dégraissant de quartz.

- Une fusaïole confectionnée dans un tesson de céramique à dégraissant calcaire.

\section{Comparaisons et ATtribution CUlturelle}

\section{Le locus 1}

Le matériel recueilli est composé principalement de produits de débitage (éclats bruts, nucléus, lamelles); la forte proportion de matériel brut évoque une aire de débitage probablement très proche. Le locus 1 est sans doute contemporain du locus 3 distant de 150 m environ, mais la pauvreté de la série ne nous permet pas de le rattacher à un stade précis du Mésolithique. Les seuls indices d'attribution au stade moyen sont un débitage de style Coincy et un fragment de pointe à retouche unilatérale.

\section{Le locus 3}

L'outillage du fonds commun présente les caractères suivants :
- Un pourcentage élevé de grattoirs (20\%), comparable au taux relevé dans le Limbourgien moyen, notamment, aux Pays-Bas, dans les séries de Oirschot V - silex (25,9\%) et Oirschot V - GQW (20,6 \%) (Rozoy, 1978). Cette importance des grattoirs sur le locus 3 s'est retrouvée plus tard dans d'autres sites du nord-Cotentin : Le Rozel "station 56 » (18\%) (Audouard 1986) ou Flamanville, Déhus (15\%) (Souffi 2000).

- Un nombre important d'éclats retouchés (19\%), comparable aux sites suivants : le Rozel "Station 56 » (23\%), Auderville, Roc de Gîte (34 \%) (Guesquière 2000) ou Flamanville «EDF»(26\%). Sur ces trois sites, les éclats minces représentent en moyenne $75 \%$ des éclats retouchés). Dans le Limbougien les éclats retouchés paraissent moins nombreux : Oirshot V - silex (11,7\%), Oirshot V - GQW (5,6\%). Sur ces deux sites les éclats minces sont également majoritaires (91\% en moyenne).

- L'absence de burin, alors que quelques exemplaires subsistent à Oirschot $\mathrm{V}$, série silex (1,9\%), et aussi à Flamanville "EDF », à Auderville, Roc de Gîte, et au Rozel. À noter d'autre part la présence d'un unique perçoir.

- Un outillage réalisé le plus souvent sur lamelles (17\%), contre $7 \%$ sur lames.

Les variations observées dans les quantités d'outils communs ne semblent pas être liées à des différences d'ordre chronologique mais plus probablement à des activités plus ou moins développées suivant les sites (fig. 14 et 15).

L'ensemble des classes d'armatures est par contre très différent de celui de Oirschot $\mathrm{V}$ où de nombreux types sont représentés. À Digulleville, il est constitué essentiellement de lamelles étroites à bord abattu (80\%), dont quelques exemplaires sont tronqués. Deux pointes à bord abattu, un fragment de pointe à retouche unilatérale et une pointe du Tardenois à base concave complètent la série. Cette prédominance des lamelles à bord abattu, le plus souvent étroites, se retrouve dans plusieurs sites du nord-Cotentin : Flamanville «EDF » (59,8 \%), Auderville, Roc de Gîte (62,8 \%), Flamanville, le Dehus (40 \%) et, dans des proportions plus faibles, à Flamanville, le Coquet $(30,4 \%)$, le Rozel «station 56 » (22,2\%). Les armatures dominantes qui y sont associées sont des pointes à base non retouchée : 29,6\% à Flamanville "EDF », 29,1 \% à Auderville, Roc de Gîte, 34,7 \% à Flamanville, le Coquet, 33,3 \% à Flamanville, le Dehus. Au Rozel «station 56 ", les triangles scalènes sont légèrement plus nombreux (fig. 16).

À Digulleville il n'a pas été trouvé d'armatures géométriques, contrairement aux principaux sites du nord-Cotentin où les triangles scalènes sont présents dans des proportions plus ou moins importantes : $6 \%$ à Flamanville «EDF », 2 à Auderville, Roc de Gîte, 30,4 \% à Flamanville, le Coquet, 10,4 \% à Flamanville, le Déhus, 24,4 \% au Rozel «station 


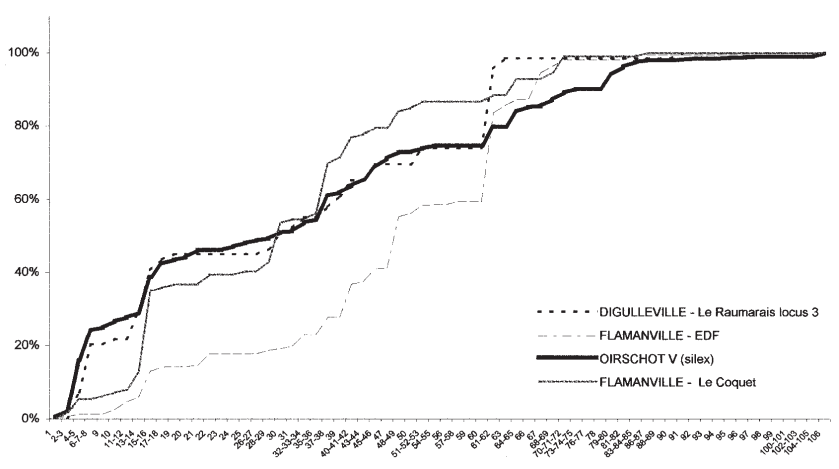

Figure 14 : proportions des outils à Digulleville, le Raumarais, locus 3 (69 outils), Flamanville « EDF » (837 outils), Flamanville, le Coquet (112 outils) et Oirschot V, série silex (418 outils). Figure 14: Tool proportions at Digulleville, le Raumarais, locus 3 (69 tools), Flamanville "EDF" (837 tools), Flamanville, le Coquet (112 tools) and Oirschot $V$, série silex (418 tools).

56 ». La présence de triangles isocèles est exceptionnelle dans cette région (3 exemplaires à Auderville sur 1251 armatures).

Les datations radiocarbone ${ }^{1}$ obtenues sur coquilles de noisettes à Flamanville «EDF " (Gif. Tan. 89334) : 8990 $\pm 180 \mathrm{BP}$, calibration à 2 sigma [8600-7600 av. J.-C.] et à Auderville (Gif. Tan. 89337) : $8460 \pm 170$ BP, cal. [80007050 av. J.-C.] confirment le positionnement des deux sites au début du Boréal, mais plusieurs centaines d'années les séparent alors que les deux séries sont très semblables (fiabilité des échantillons?).

Dans le Finistère, Le site de Toul-an-Naouc'h à Plougoulm, attribué au groupe dit à industrie de type Bertheaume (Berrou et Gouletquer 1973), est caractérisé par des armatures pygmées connues ailleurs dans le Sauveterrien (triangles de Monclus, lamelles à bord abattu, pointes triangulaires effilées y dominent, mais les pointes de Sauveterre y sont absentes). On a pu y étudier 2 séries pour un total de 29 armatures. Une datation sur coquille de noisette a pu être obtenue (Gif. Tan. 89339) : $8830 \pm 180$ BP, cal. [83507500 av. J.-C.]. L'industrie, tout d'abord considérée comme pouvant être un faciès régional du techno-complexe sauveterrien (Kayser et al. 1990), a fait l'objet d'un nouvel examen (Blanchet et al. 2006). Les différences observées entre les deux groupes portent sur la variété des types de pointes dans le groupe breton et sur l'absence de pointes de Sauveterre. Le groupe de Bertheaume ne serait pas l'extension de la culture Sauveterrienne; la nouvelle hypothèse est celle d'une convergence technique entre groupes qui évolueraient dans les grandes lignes de la même façon.

Dans la Somme, où le Mésolithique est bien documenté (Ducrocq 2001), les gisements datés du début du Boréal ont

1. Les datations radiocarbone ont été calibrées par P. Sabatier d'après la courbe atmosphérique de Reimer et al. 2004, logiciel Oxcal - v310.

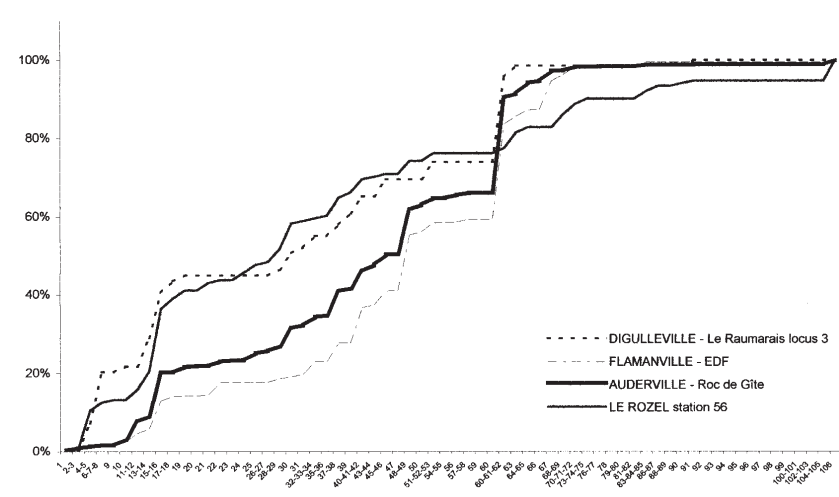

Figure 15 : proportions des outils à Digulleville, le Raumarais, locus 3 (69 outils), Flamanville "EDF » (837 outils), Auderville, Roc de Gîte (2519 outils) et Le Rozel "Station 56 " (146 outils).

Figure 15: Tool proportions at Digulleville, le Raumarais, locus $3(69$ tools), Flamanville "EDF" (837 tools), Auderville, Roc de Gîte (2519 tools) and Le Rozel "Station 56" (146 tools).

des assemblages d'armatures dominés par les segments et les pointes à base retouchée :

- Hangest-sur-Somme, gravière II nord : (Gif. 8912) : $8830 \pm 90 \mathrm{BP}$, calibration à 2 sigma [8250-7 650 av. J.-C.] (sur noisettes);

- Saleux, Vierge Catherine, niv. inférieur (Lyon. 78-Oxa 4929) : $8645 \pm 70$ BP, cal. [7940-7540 av. J.-C.] (sur os).

Dans la même région, des gisements à lamelles à dos dominantes, associées à des microlithes variés (dont des armatures à retouches couvrantes), sont datés de la deuxième partie du Boréal :

- Hangest-sur-Somme, gravière III 2/3 (Gif. 9276) : 8290 \pm 70 BP, cal. [7 520-7 140 av. J.-C.] (sur noisettes);

- La Chaussée-Tirancourt, le Petit Marais, fosse 3 (Gif. 9908) : $8180 \pm 70$ BP, cal. [7450-7 040 av. J.-C.] (sur noisettes).

Des ensembles comparables existent en Belgique, en particulier dans le Groupe de Sonnisse Heide (Sites de Helchteren - Sonnisse Heide II, Kruishouten Kerkakkers, Weelde I, où l'élément le plus caractéristique est la prédominance des lamelles à bord abattu (80 à $90 \%$ ), en majorité étroites. Elles sont généralement accompagnées par un nombre variable de pointes à base retouchée, type pointe $\mathrm{du}$ Tardenois et/ou de microlithes à retouches couvrantes. Les segments, triangles et pointes à base non retouchée sont rares. (Crombé 1999). Les datations obtenues situeraient ce groupe dans la seconde moitié du Boréal :

- Kruishoutem (Utc. 2023) : $8270 \pm 80$ BP, cal. [7 5007080 av. J.-C.] (sur noisettes);

- Weelde I (Oxa. 141) : $8200 \pm 150$ BP, cal. [7 550-6700 av. J.-C.] (sur noisettes). 


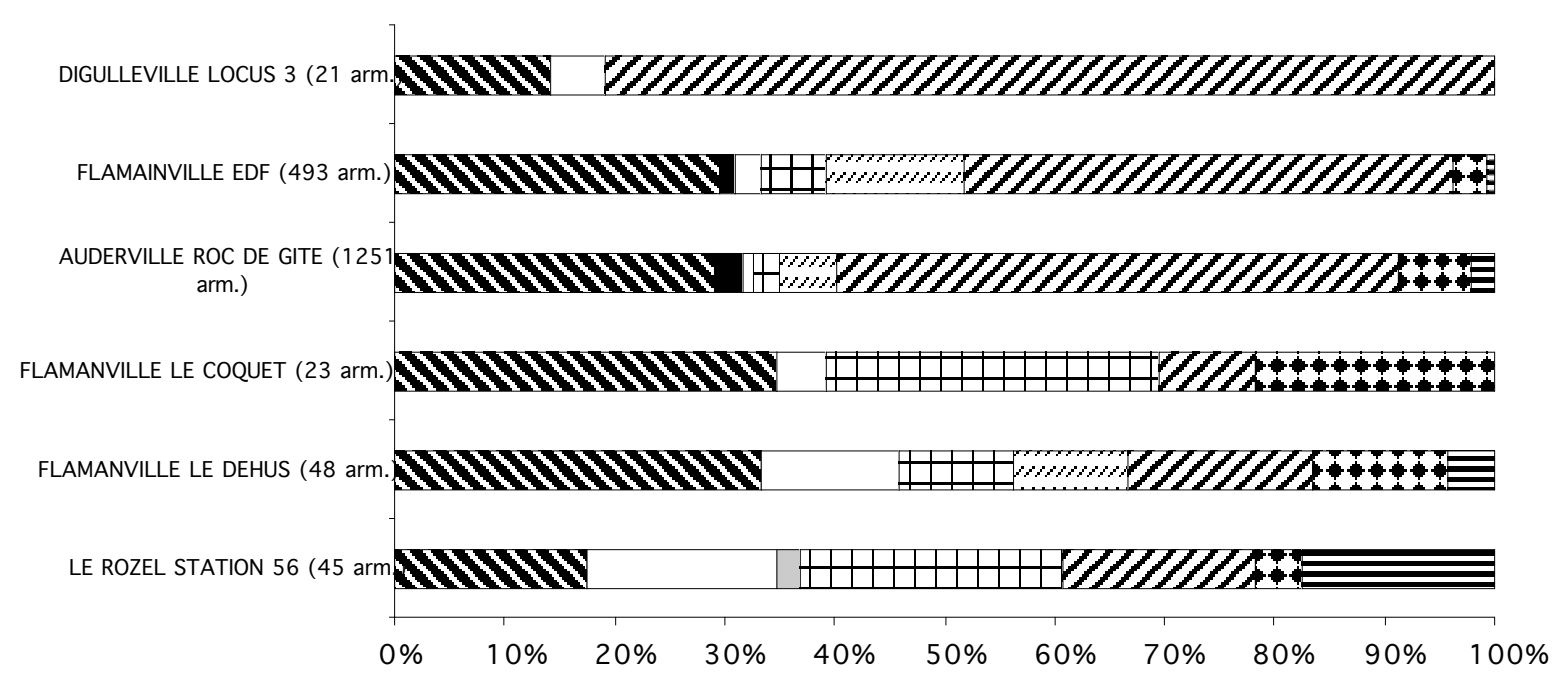

\begin{tabular}{|ll|}
\hline Pointes à base non retouchée & $\square$ Segments \\
$\square$ Pointes à base retouchée & $\square$ Triangles scalènes \\
$\square$ Lamelles scalènes & $\square$ Lamelles étroites à bord abattu \\
Lamelles à bord abattu & $\Xi$ Divers \\
\hline
\end{tabular}

Figure 16 : proportions des armatures à Digulleville, le Raumarais, locus 3, Auderville, Roc de Gîte, Flamanvillle "EDF », Flamanville, le Coquet, Flamanville, le Dehus et Le Rozel «station 56 ».

Figure 16: Proportions of armatures at Digulleville, le Raumarais, locus 3, Auderville, Roc de Gîte, Flamanvillle "EDF", Flamanville, le Coquet, Flamanville, le Dehus and Le Rozel "station 56".

\section{ConClusion}

Il y a un peu plus de vingt ans, lorsque l'un de nous avait réalisé cette étude, la documentation disponible sur le Mésolithique normand était des plus pauvres et l'industrie de Digulleville n'avait pu être rattachée à aucun des rares gisements connus à l'époque en Normandie : Montfarville, la Houe (Michel 1974), Saint-Wandrille-Rançon (Verron 1977; Chancerel 1983). Aucune série semblable n’avait été trouvée dans les différents groupes reconnus en France, la série de Digulleville se distinguant notamment par l'abondance des grattoirs et la prédominance des lamelles étroites à bord abattu. La similitude de composition de l'outillage $\mathrm{du}$ fonds commun avec le Limbourgien moyen, le taux relativement important des armatures, enfin l'absence de trapèzes, furent les principaux critères d'attribution du gisement au début du stade moyen du Mésolithique. L'hypothèse émise à l'époque était que le locus 3 pouvait appartenir à un groupe autonome de population en raison de l'originalité de composition de son outillage, mais la faible quantité de matériel récolté amenait des réserves et rendait nécessaire la fouille et l'étude de nouvelles stations pour éventuellement le confirmer. Les études publiées récemment par P. Lefèvre (Ghesquière et al 2000) sur les séries lithiques de Flamanville
«EDF» (Fouilles Y. Roupin) et par E. Ghesquière (Ghesquière et al. 2000) à Auderville, Roc de Gîte (fouilles A. Chancerel), attribuées au début du Mésolithique moyen, montrent de nombreux points communs avec le locus 3 de Digulleville (fig. 14 à 16). Cependant, la série de Digulleville se démarque pour l'instant des autres sites du nord-Cotentin par la prédominance des lamelles étroites à bord abattu et l'absence d'armatures géométriques. Son attribution au début du Mésolithique moyen est à proposer avec prudence compte tenu du nombre restreint d'armatures et des exemples de la Somme et de la Belgique où des sites à forte proportion de lamelles étroites à bord abattu sont datés de la deuxième moitié du Boréal.

Dans une synthèse récente (Ghesquière et al. 2003), un peu moins d'une trentaine de sites ont été pris en compte pour la définition du Mésolithique moyen du nord-Cotentin, mais les séries sont très inégales. $98 \%$ des armatures proviennent des six gisements que nous avons retenus (fig. 16), seuls quatre sites ont fait l'objet de fouilles ou de sondages et on ne dispose que de deux datations ${ }^{14} \mathrm{C}$. Il est donc nécessaire qu'elles soient multipliées pour parvenir à établir une chronologie de référence. Ceci permettrait peut-être de positionner plus précisément la très petite série de Digulleville par comparaison des assemblages lithiques, les prélèvements de charbons effec- 
tués en vue de datation radiocarbone étant malheureusement perdus. À en juger par la faible densité de matériel mis au jour et la localisation des concentrations, le site du Raumarais semble correspondre à des occupations ponctuelles, peut-être saisonnières, de petits groupes de chasseurs, plutôt qu'à un campement de base. Le comblement apparemment rapide de la cuvette du foyer après son utilisation renforce cette hypothèse. D'autres traces d'installations similaires existent sans doute dans les environs immédiats mais dorment encore sous la lande, probablement disséminées sur une grande surface. Peut-être seront elles découvertes un jour à l'occasion de nouveaux aménagements.

\section{Remerciements}

Nous tenons à remercier tous les fouilleurs : Laurent Attinault, Anne Battistoni, Xavier Guillard, Thierry Leboulanger, Patrick Lebret, Annie Philippon, Marie-Jeanne Roche, Béatrice Simon, ainsi que Marie Françoise Goude et Christophe Toupet pour leur contribution à la recherche bibliographique.

\section{Bibliographie}

Audouard, M., 1986 - L'occupation mésolithique au Rozel (Manche) : la station 56, Revue archéologique de l'Ouest, 3, p. 5-21.

Berrou, P. et Gouletquer, P.-L., 1973 -L'Épipaléolithique de la région de Plovan (Finistère). Note préliminaire, Bulletin de la Société préhistorique française, 70, 6, p. 166-172.

Blanchet, S., Kayser, O., Marchand, G. et Yven, E., 2006 - Le Mésolithique moyen en Finistère : de nouvelles datations pour le groupe de Bertheaume, Bulletin de la Société préhistorique française, 103, 3, p. 507-517.

Chancerel, A., 1983 - La série mésolithique de Saint-WandrilleRançon (Seine maritime), Bulletin de la Société préhistorique française, 80, 10/12, p. 335-348.

Chancerel, A. et Paulet-Locadr, M.-A., 1991 - Le Mésolithique en Normandie: état des recherches, Mésolithique et Néolithisation en France et dans les régions limitrophes $\left(113^{\mathrm{e}}\right.$ congrès national des Sociétés savantes, Strasbourg), Paris, CTHS, p. 213-229.

Crombé, P., 1999 - Vers une nouvelle chronologie absolue pour le Mésolithique en Belgique, in P. Bintz (dir.) et A. THÉvenin (éd.), l'Europe des derniers chasseurs : Épipaléolithique et Mésolithique en Europe, ( $5^{\mathrm{e}}$ colloque international UISPP - Commission XII, Grenoble 1995), p. 189-199.

Ducroce, T., 2001 - Le Mésolithique du bassin de la Somme, Lille, Université des Sciences et Technologies (publications du CERP, n $\left.{ }^{\circ} 7\right), 255$ p.
Ghesquière, E., 2000 - Le site d'Auderville-Roc de Gîte, in E. Ghesquière, P, Lefèvre, C. Marcigny et B. Souffi, Le Mésolithique moyen du nord-Cotentin, Basse-Normandie, France, Oxford, Hadrian Books (BAR International Series 856), p. 6-163.

Ghesquière, E., Lefèvre, P., Marcigny, C. et al., 2003 - Le Mésolithique moyen du nord-Cotentin (Manche), Bulletin de la Société préhistorique française, 100, 4, p. 649-670.

Groupe D’ÉTude de L'ÉPIPALÉOLITHIQUe-MésolithiQue (GEEM), 1969 - Épipaléolithique-Mésolithique, les microlithes géométriques, Bulletin de la Société préhistorique française, 66, p. 355-366.

Groupe D’Étude de l'ÉPIPALÉOLITHIQUe-MésolithiQue (GEEM), 1972 - Épipaléolithique-Mésolithique, les armatures non géométriques 1, Bulletin de la Société préhistorique française, 69, 1, p. 364-375.

Groupe D'ÉTUde DE L'ÉPIPALÉolithiQUe-MésolithiQue (GEEM), 1975 - Épipaléolithique-Mésolithique, l'outillage du fonds commun. 1 - Grattoirs - éclats retouchés - burins - perçoirs, Bulletin de la Société préhistorique française, 72, p. 319-332.

Inizan, M.- L., Reduron, M., Roche, H. et Tixier, J., 1995 - Préhistoire de la pierre taillée, (t. 4, Technologie de la pierre taillée), CREP, 199 p., 79 fig.

Kayser, O., Le Goff, J.-C. et RouÉ, D., 1990 - Le site mésolithique de Toul-an-Naouc'h, Plougoulm (Finistère), Revue archéologique de l'Ouest, 7, p. 23-29.

Lefèvre, P., 2000 - Le site de Flamanville - Centrale EDF, in E. Ghesquière, P. Lefèvre, C. Marcigny et B. Souffi, Le Mésolithique moyen du nord-Cotentin, Basse-Normandie, France, Oxford, Hadrian Books (BAR International Series 856), p. 164-200.

Leroi-Gourhan, A. et al., 1966 - la Préhistoire, Paris, PUF, coll. "Nouvelle Clio ", 366 p.

Leroy, D., 1993 - Sondages sur le site mésolithique du Coquet à Flamanville (Manche), Revue archéologique de l'Ouest, 10, p. 9-17.

LetTerlé, F. et Verron, G., 1986 - Un site d'habitat campaniforme à Digulleville (Manche), Revue archéologique de l'Ouest, suppl. 1 (actes du X colloque interrégional sur le Néolithique, Caen 1983), p. 237-252.

Michel, D., 1974 - Une nouvelle station préhistorique au lieu-dit "la Houe ", commune de Montfarville (Manche), Mémoires de la Société nationale des Sciences naturelles et mathématiques de Cherbourg (1969-1970), p. 9-69.

Rozoy, Dr. J.-G., 1967a - Essai d'adaptation des méthodes statistiques à l'Épipaléolithique ("Mésolithique»). Liste-type provisoire et premiers résultats, Bulletin de la Société préhistorique française, 64, 1, p. 209-226.

Rozoy, Dr. J.-G., 1967b - Typologie de l'Épipaléolithique franco-Belge, Bulletin de la Société préhistorique française, 64, 1, p. $227-260$. 
Rozoy, Dr. J.-G., 1968a - Typologie de l'Épipaléolithique (Mésolithique) fran co-Belge, Bulletin de la Société préhistorique française, 65, 1, p. 335-364.

Rozoy, Dr. J.-G., 1968b - L'étude du matériel brut et des microburins dans l'Épipaléolithique (Mésolithique) francoBelge, Bulletin de la Société préhistorique française, 65, 1, p. 365-390.

Rozor, Dr. J.-G., 1971 - Tardenoisien et Sauveterrien, Bulletin de la Société préhistorique française, 68, 1, p. 345-374.

Rozor, Dr. J.-G., 1978 - Les derniers chasseurs. L'Épipaléolithique en France et en Belgique, essai de synthèse, Bulletin de la Société archéologique Champenoise ( $\mathrm{n}^{\circ}$ spécial, 3 tomes), 1260 p., $259 \mathrm{pl}$.

Soufri, B., 2000. - Le site de Flamanville - Le Déhus, in E. Ghesquière, P. Lefèvre, C. Marcigny, et B. Souffi, Le Mésolithique moyen du nord-Cotentin, Basse-Normandie,
France, Oxford, Hadrian Books (BAR International Series 856), p. 201-221.

Verron, G., 1975 - Informations archéologiques, circonscription de haute et basse Normandie, Gallia préhistoire, 18, 2, p. 471-510.

Verron, G., 1977 - Informations archéologiques, circonscription de haute et basse Normandie, Gallia préhistoire, 20, 2, p. 357-406.

VerRon, G., 1981 - Informations archéologiques, circonscription de haute et basse Normandie, Gallia préhistoire, 24, 2, p. 365-394. 